\title{
Etude hydrogéologique des séries triasiques dans la région de Jeffara-Dahar (Sud Tunisien): Apport des diagraphies et de la sismique réflexion
}

\section{Hydrogeological study of the Triassic series in the Jeffara- Dahar region (Southern part of Tunisia): Contribution of well logs data and seismic reflection}

\author{
R. Ben Lasmar ${ }^{1}$, R. Guellala ${ }^{1,2}$, L. Zouhri ${ }^{3}$ B. Sarsar Naouali ${ }^{1,4}$, M. Garrach ${ }^{5}$ \& M.H. Inoubli ${ }^{1}$ \\ 1 Département de Géologie, Faculté des Sciences de Tunis, Unité de Recherches de Géophysique Appliquée aux Matériaux \\ et aux Minerais (URGAMM), 2092, El Manar, Tunisie. Email: benlasmar.rafika@hotmail.fr \\ 2 Laboratoire de Géoressources, CERTE, Technopôle de Borj Cedria, Tunisie \\ 3 HydrISE, Institut Polytechnique La Salle Beauvais, 19 Rue Pierre Waguet, 60026 Beauvais, France \\ 4 Entreprise Tunisienne d'Activité Pétrolière (ETAP), Charguia II, Tunisie \\ 5 Commissariat Régional de Développement Agricole de Tataouine
}

\section{RÉSUMÉ}

La présente étude concerne l'interprétation des données de la sismique réflexion et celles des puits y compris les enregistrements diagraphiques en vue d'une meilleure caractérisation de l'aquifère triasique de la région de Jeffara-Dahar (Sud-Est de la Tunisie) qui constitue une cible potentielle pour l'alimentation en eau.

Les logs lithologiques associés aux enregistrements diagraphiques correspondants montrent que les principaux réservoirs sont les grés de Sidi Stout, Kirchaou et Touareg ainsi que les dolomies de Mekraneb et Rehach. L'interprétation diagraphique met en évidence des niveaux de faible perméabilité et d'autres perméables et bien fracturées qui pourraient jouer un rôle dans l'acheminement des eaux souterraines.

Les profils sismiques interprétés et la cartographie élaborée en conséquence mettent en évidence que l'aquifère triasique est compartimenté en blocs soulevés et affaissés. Cette structuration, contrôlée par la réactivation des accidents E-W et NW-SE en failles normales au cours du Trias, influence l'épaisseur des formations perméables ainsi que le sens d'écoulement de la nappe d'eau.

Les résultats obtenus seront utiles pour la rationalisation des futures recherches hydrogéologiques dans la région de Jeffara - Dahar.

Mots clefs: Sud Tunisien; Jeffara-Dahar; aquifère triasique; diagraphies; sismique réflexion.

\section{ABSTRACT}

The present study concentrates on the interpretation of well logs and seismic reflection data in the JeffaraDahar region (Southeast part of Tunisia) for a better characterization of the Triassic aquifer, a potential target of water supply. Lithological columns and their corresponding well logs reveal that Sidi Stout, Kirchaou and Touareg

Recibido el 20 de abril de 2015 / Aceptado el 18 de diciembre de 2015 / Publicado online el 3 de marzo de 2016

Citation / Cómo citar este artículo: R. Ben Lasmar, et al. (2016). Etude hydrogéologique des séries triasiques dans la région de Jeffara-Dahar (Sud Tunisien): Apport des diagraphies et de la sismique réflexion. Estudios Geológicos 72(1): e044. http://dx.doi. org/10.3989/egeol.42114.373.

Copyright: () 2016 CSIC. This is an open-access article distributed under the terms of the Creative Commons Attribution-Non Commercial (by-nc) Spain 3.0 License. 
sandstones as well as Mekraneb and Rehach dolomites are the main reservoirs of the Triassic aquifer. Well log analysis highlights many permeable and fractured layers that could play an important role in the groundwater circulation.

The interpreted seismic sections and the resulting isochrone maps show a tectonic influence on the Triassic aquifer geometry in the Jeffara-Dahar region. The normal faulting of E-W and NW-SE accidents created an aquifer compartmentalized by raised and tilted blocks. Seismic cross-sections reveal that this structure controls the depth of permeable formations and the circulation of groundwater.

These results will be useful for rationalising the future hydrogeological research that will be undertaken in the Jeffara-Dahar area.

Keywords: Tunisia; Jeffara-Dahar area; Triassic aquifer; well logs; Seismic reflection.

\section{Introduction}

Les séries triasiques étaient depuis longtemps l'objet de nombreuses études d'ordre stratigraphique (Cuif \& Gautret, 1987; Gautret, 1991; Peybernes et al., 1993; Gand et al., 2000; Brayard et al., 2007; Pineiro, 2012), structural (Perthuisot, 1983, Lemaire et al., 1998; Bobier et al., 1991; Chikhaoui et al., 2002; Khomsi,2004; Gharbi et al., 2005; Jallouli et al., 2005; Ben Chalbi et al., 2006; Roger et al., 2008), et minéralogique (Hartmann, 1963; Vila et al., 1998; Rossi, 2003; Hoa, 2008; Li, 2011; Oliver et al., 2014). Ce sont essentiellement les intérêts miniers et pétroliers (Mennig,1963; Espitalié et al., 1988; Alem et al. 1998; Jamal et al., 2000; Acheche et al., 2001; Naouali Sarsar et al., 2013) qui ont été visés. Les potentialités hydrogéologiques de ces séries sont rarement évoqués (Rebeix et al., 2011).

La présente étude intéresse les dépôts triasiques de la région de Jeffara-Dahar qui renferment une intéressante nappe d'eau qui fait partie d'un immense système aquifère couvrant le domaine saharien Algéro-TunisoLybien (UNESCO, 1972; OSS, 2003).

La région de Jeffara-Dahar couvre la partie NordEst de la Tunisie méridionale qui correspond à une zone aride où la pluviométrie moyenne annuelle est de $100 \mathrm{~mm} / \mathrm{an}$ (DGRE, 2013) et les ressources en eau sont essentiellement souterraines. Le réchauffement climatique, la population croissante et l'accroissement des demandes en eau, particulièrement dans les secteurs touristique et agricole réduiront inévitablement ces ressources. Pour pallier à cette situation toute étude portant sur la protection et l'amélioration de la gestion des nappes d'eau du Sud tunisien est encouragée par l'Etat.

Dans la région de Jeffara-Dahar, l'exploitation des réserves de la nappe du Trias contribuera surement à l'atténuation de la pénurie en eau. Cependant les connaissances géologiques et hydrogéologiques sont trop fragmentaires pour proposer des zones et des stratégies d'exploitation de cette nappe. Ce travail vise à préciser la lithologie des séries triasiques ainsi que leur géométrie et son impact sur le fonctionnement hydrogéologique du système aquifère logé dans ces séries, comme base préalable à l'élaboration d'un modèle hydrogéologique le mieux adapté à la région.

Généralement, la prospection des nappes d'eau est le domaine d'application privilégié de la méthode électrique (Meyer de Stadelhofen, 1991; Gasmi, 2002; Koussoubé et al., 2003; Zouhri et al., 2004; Gouasmia et al., 2006; Asfahani, 2006; Guellala et al., 2009; 2014). Vue que les séries triasiques atteignent des grandes profondeurs, la méthode de sismique réflexion apparait plus intéressante pour une meilleure caractérisation de ces formations (Astier, 1971; Jaffal et al., 2002; Zouhri et al., 2003; Larroque \& Dupuy, 2004; Sumanovac, 2006; Lachaal et al., 2011; Gabtni et al., 2012).

\section{Contexte géologique}

En Tunisie, la plateforme saharienne (Fig.1a) est caractérisée par trois différents domaines qui sont d'Est en Ouest (Fig. 1b): Jeffara, Dahar et Erg Oriental.

La Jeffara est une vaste plaine où affleurent uniquement les dépôts mio-plio-quaternaires (Ben Ayed \& Khessibi, 1981; Bouaziz, 1995). Elle est limitée à l'Ouest par un accident majeur orienté NW-SE dénommé l'accident de Mednine (Castany, 1954) ou de Jeffara (Duvernoy, 1994).

Le Dahar est une large structure monoclinale plongeant de 1 à $3^{\circ}$ vers l'Ouest (Ben Ismail, 1991). Dans ce domaine, les séries mésozoïques sont largement exposées. Dans sa partie septentrionale, se trouve l'affleurement du Permien supérieur du Jebel Tebaga de Medenine (Berkaloff, 1933; Mathieu, 1949; 


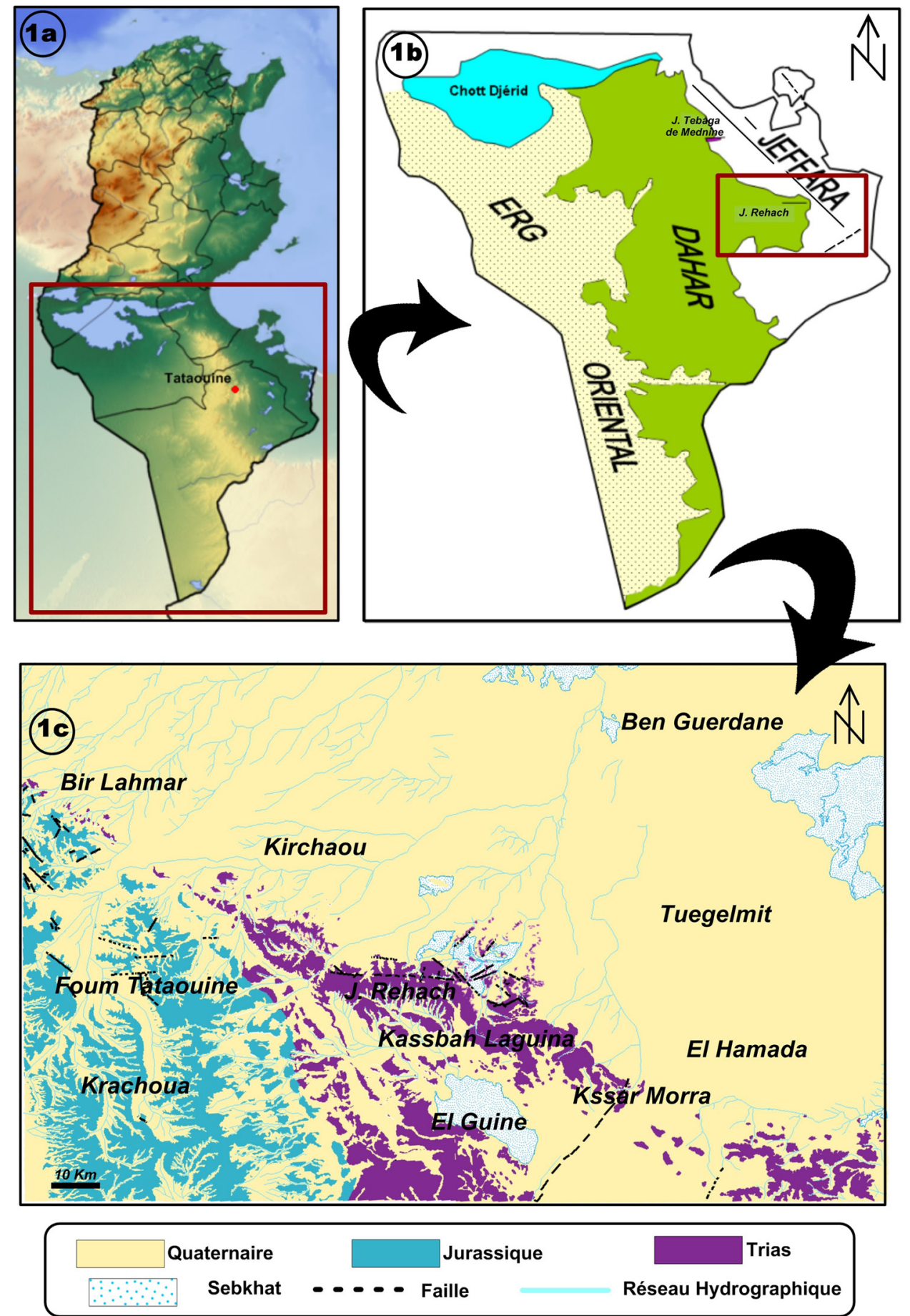

Figure 1.-Secteur d'étude. 1a. Localisation. 2a. Cadre géographique. 3a. Carte géologique.

Glintzboeckel \& Rabaté 1964; Baird, 1967; Newell et al., 1976; Termier et al., 1977; Khessibi, 1985; Chaouahi, 1988; M'Rabet et al., 1987; Razghallah et al., 1989; Raulin et al., 2011).
L'Erg oriental est un espace désertique couvert par des dunes (Ben Ismail, 1991).

Notre secteur d'étude occupe une position charnière entre le domaine de la Jeffara et celui du Dahar. 
Il est caractérisé par des affleurements d'âge Trias, Jurassique et Quaternaire (Fig. 1c).

Les dépôts triasiques constituant notre objet d'étude sont largement étendus dans le Sud-Est tunisien. Une série stratigraphique complète est identifiée le long d'une ceinture NW-SE s'étendant du Jebel Tebaga de Mednine à la frontière libyenne. Le Trias inférieur à moyen est représenté par les grés de Sidi Stout (Scythien-Anisien) et les grés de Kirchaou (Ladinien),
Le Trias supérieur est marqué par les dolomies de Mekraneb (Carnien inférieur), les grés de Touareg (Carnien moyen), les dolomies de Rehach (Carnien supérieur) (Burollet, 1963; Busson, 1970, 1972; Ben Ismail, 1991; Bouaziz, 1995), les argiles et les gypses de Mhira (Carnien supérieur-Norien inférieur), les dolomies de Massoudi (Norien-Réthien) et les évaporites de Bhir (Trias supérieur p.p -Sinemurien) (Bouaziz, 1995; Kamoun et al., 2001) (Fig. 2).

\begin{tabular}{|c|c|c|c|}
\hline \multicolumn{3}{|c|}{ Chronostratigraphie } & Log \\
\hline \multirow{6}{*}{ 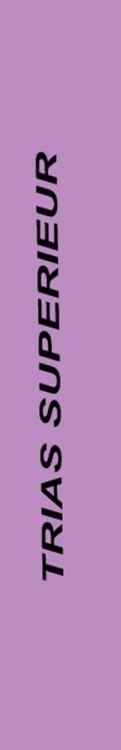 } & Trias supérieur-Sinemurien & Les évaporites de Bhir & \\
\hline & Norien-Réthien & Les dolomies de Massoudi & $\frac{1}{1}$ \\
\hline & Carnien supérieur-Norien inférieur & Les argiles et les gypses de Mhira & \\
\hline & Carnien supérieur & Les dolomies de Rehach & \begin{tabular}{|l|l|}
1 & \\
1 &
\end{tabular} \\
\hline & Carnien moyen & Les grés de Touareg & $\because$ \\
\hline & Carnien inférieur & Les dolomies de Mekraneb & 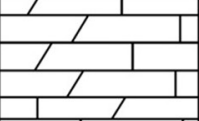 \\
\hline \multirow{2}{*}{ 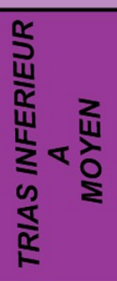 } & Ladinien & Les grés de Kirchaou & 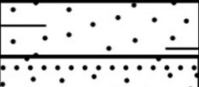 \\
\hline & Scythien-Anisien & Les grés de Sidi Toui & $\because \because \because$ \\
\hline \multicolumn{3}{|c|}{ Permien } & \begin{tabular}{|l|l|l|} 
& & \\
& & \\
& & \\
\end{tabular} \\
\hline
\end{tabular}

Légende:

\begin{tabular}{|c|c|c|c|}
\hline $\begin{array}{l}1 \\
\end{array}$ Calcaire & $\because \because \because$ Grés & $\because \dot{ }$ & Sable argileux \\
\hline Gypse et Anhydrite & Argile & 7 & Dolomie \\
\hline
\end{tabular}

Figure 2.-Colonne lithologique synthétique des séries triasiques. 


\section{Contexte hydrogéologique}

Les nappes du Sud tunisien font partie du système aquifère multicouche couvrant le Sahara Septentrional de l'A frique du Nord (SASS). Les formations perméables du Trias (Bundstandstein) constituent le niveau aquifère le plus ancien (OSS, 2003). Les carbonates du Jurassique moyen et supérieur représentent un réservoir de moindre importance. Les séries sablo-gréseuses du Crétacé inférieur logent l'aquifère du Continental Intercalaire (Guellala et al., 2011). Les formations carbonatées du Turonien et du Sénonien et les formations détritiques du Miocène (UNESCO, 1972; OSS, 2003; Abid, 2010) renferment la nappe du Complexe Terminal.

Ces différents aquifères sont séparés par des couches imperméables ou semi-perméables. Les évaporites du Trias supérieur -Jurassique inférieur, les argiles du Cénomanien et celles du Mio-Pliocène sont les principaux aquicludes.

Plusieurs études hydrogéologiques ayant pour objet les aquifères du Continental Intercalaire et du Complexe Terminal dans le Sud tunisien (Besbes \&
Ben Ammar, 1985; Mansouri, 1988; Mamou, 1990; Zammouri, 1990; Yahyaoui, 1996; Chalbaoui \& Ben Dhia, 2004; Guellala et al., 2011; Gabtni et al., 2012) et dans l'ensemble du Sahara Septentrional (UNESCO, 1972, OSS, 2003, Ould Baba Sy, 2005) ont été réalisées. L'aquifère du Jurassique (Garrach, 2005) et particulièrement celui du Trias ont été négligés.

Le Trias, essentiellement argileux et salifère dans le bassin saharien algérien, n'a pas d'intérêt hydrogéologique. En Lybie, le calcaire dolomitique de la formation Azizia (Trias supérieur (Carnien)) bien fracturé, constitue un bon aquifère (OSS, 2003). Dans le Sud tunisien, les grés et les dolomies du Trias inférieur et moyen sont investigués.

Dans la région de la Jeffara-Dahar, la nappe du Trias est peu exploitée; le nombre des forages d'eau ne dépasse pas 50 malgré l'abondance des affleurements triasiques et qu'elle soit reconnue depuis 1970 (Busson, 1970).

Les mesures piézométriques de cette nappe relatives à l'année 2014 montrent une nette diminution des valeurs en allant de l'Ouest vers l'Est indiquant un écoulement des eaux souterraines dans cette direction (Fig. 3).

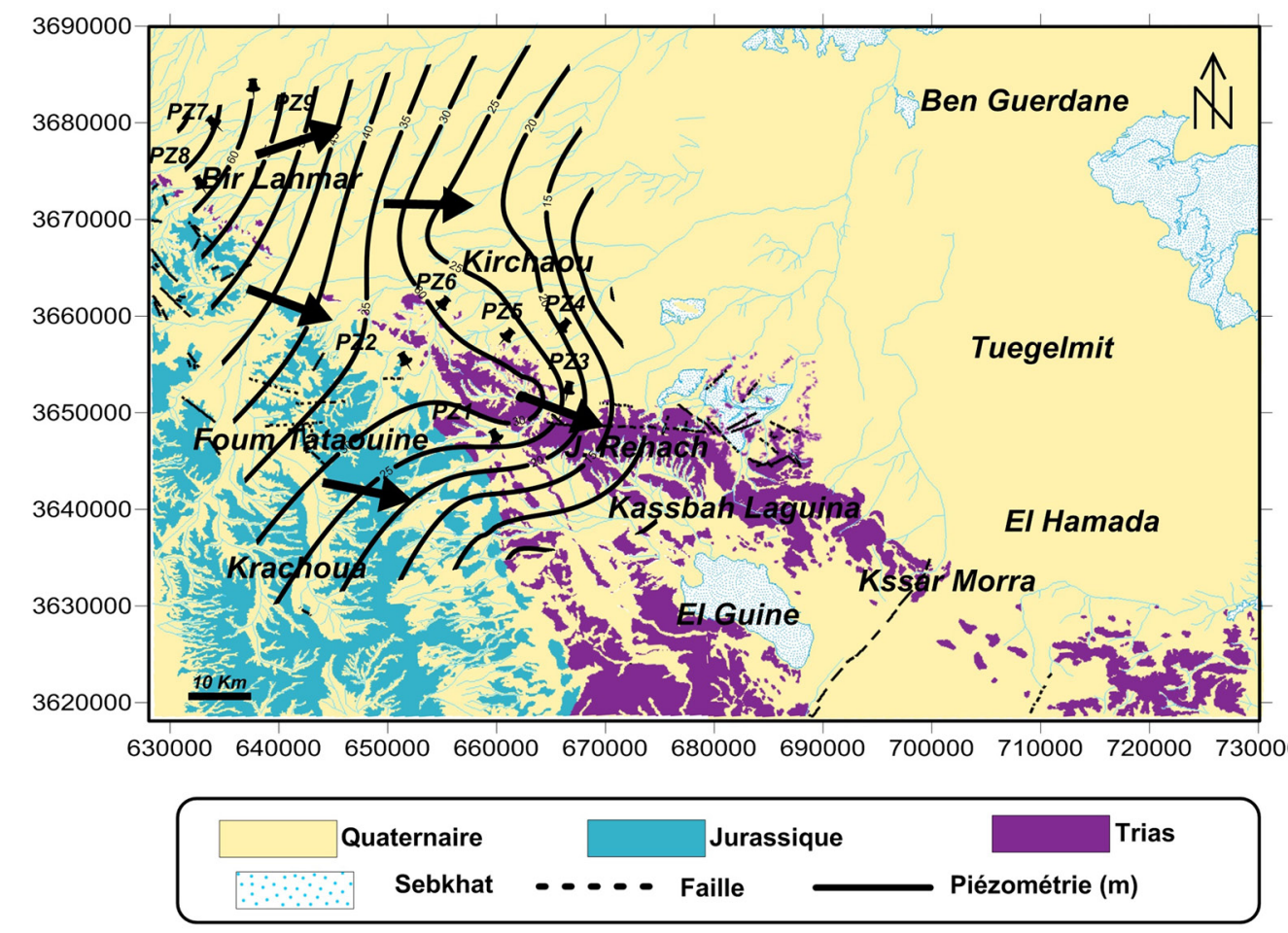

Figure 3.-Carte piézométrique du Trias gréseux. 
La carte de salinité établie pour les niveaux gréseux du Trias (Fig. 4) révèle des valeurs allant de $0.8 \mathrm{~g} / 1$ à $4.7 \mathrm{~g} / \mathrm{l}$. Elles augmentent de l'Ouest vers l'Est avec une parfaite concordance avec le sens d'écoulement. Pour les niveaux aquifères dolomitiques, les mesures sont insuffisantes pour réaliser une carte. Les valeurs disponibles varient entre $3.5 \mathrm{~g} / 1$ et $4.5 \mathrm{~g} / 1$.

\section{Données et méthodes}

La présente étude est basée sur des données de puits, des enregistrements diagraphiques et des profils sismiques (Fig. 5). Ces données sont fournies par la Direction Générale des Ressources en Eau (DGRE Tunisie) et l'Entreprise Tunisienne d'Activités Pétrolières (ETAP).

\section{Puits}

Les puits consultés consistent en des puits pétroliers et des sondages d'eau captant l'aquifère triasique. Ces puits constituent les principaux éléments de calage des données géophysiques. Additionnellement, leurs corrélations permettent de déceler les variations latérales et verticales des niveaux réservoirs.

La majorité des puits pétroliers atteignent d'importantes côtes; les puits «A» et «B» sont respectivement profonds de $3800 \mathrm{~m}$ et $2265 \mathrm{~m}$. Ils recoupent les séries quaternaires, néogènes, paléogènes, crétacées, jurassiques et triasiques. La plupart des sondages d'eau sont profonds de moins de $1000 \mathrm{~m}$ et ne présentent que des dépôts quaternaires, néogènes et une partie de la série triasique. Cependant, les caractéristiques hydrogéologiques et physico-chimiques de l'aquifère triasique sont déterminées à partir des mesures faites au niveau de ces sondages.

Ainsi, pour garantir une meilleure caractérisation de l'aquifère triasique, nous avons utilisé conjointement les données des puits pétroliers et ceux des sondages d'eau.

\section{Diagraphies}

Les déblais brassés de boue de forage sont les seuls témoins des colonnes lithologiques des puits disponibles. Dans ces conditions, il est difficile de bien délimiter les niveaux réservoirs. Pour pallier à

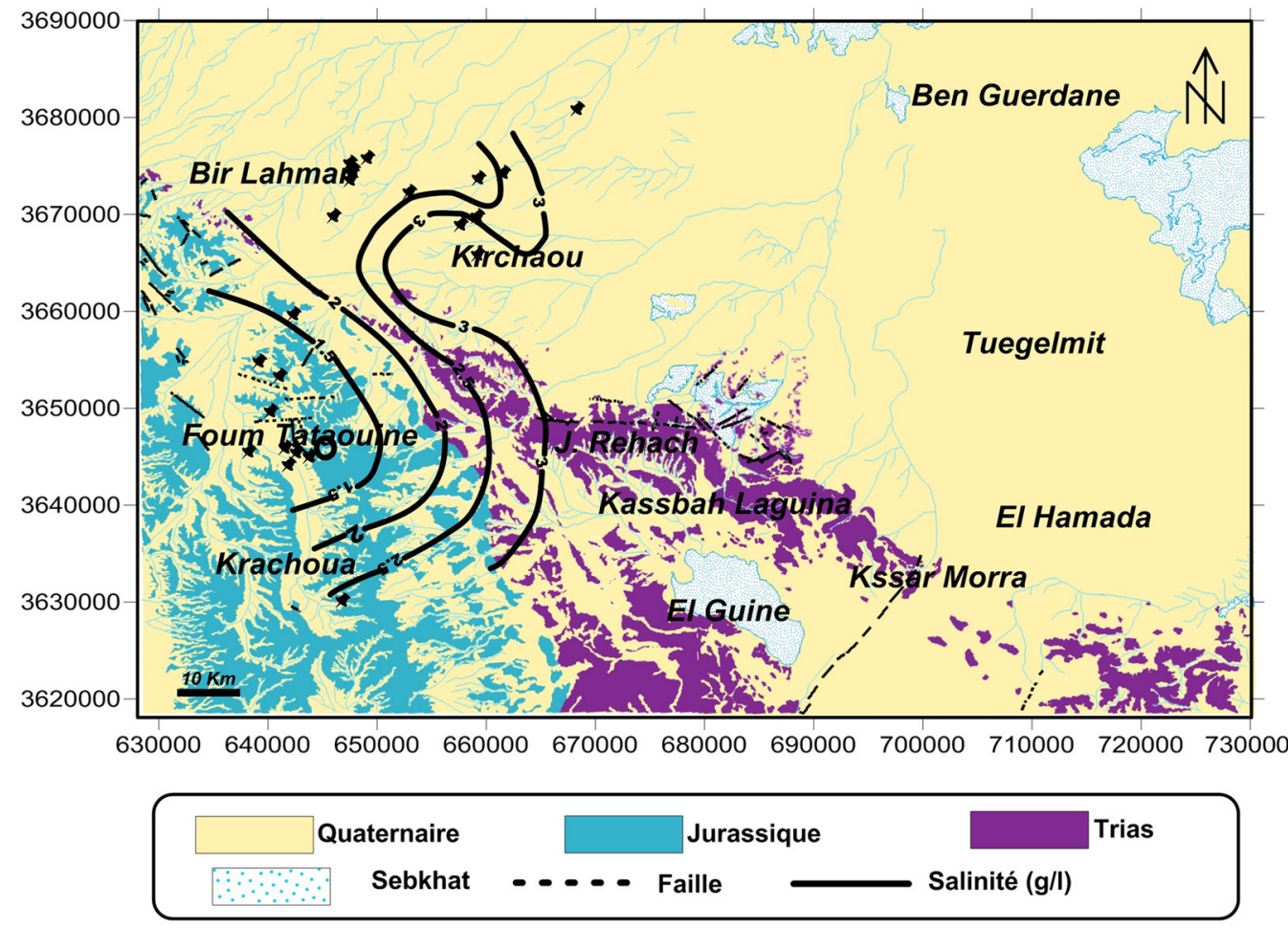

Figure 4.-Carte de salinité des niveaux gréseux triasiques. 
ces inconvénients, nous avons eu recours aux diagraphies qui sont des enregistrements continus d'un ou plusieurs paramètres physiques en fonction de la profondeur.

Les diagraphies réalisées dans les forages d'eau disponibles, sont le Gamma Ray et la résistivité.

L'émission des rayons gamma est tributaire de la présence d'éléments radioactifs (potassium K40, thorium th 232, Uranium U238) dans les séries sédimentaires. Les argiles qui constituent le support préférentiel de ces éléments ont une forte radioactivité naturelle variant entre 100 et 200 API (Chappelier, 1992).

La résistivité est le paramètre le plus mesuré en diagraphies électriques. Plusieurs dispositifs de mesure ayant différents rayons d'investigation sont utilisés. Les logs de résistivité disponibles sont enregistrés par les sondes petite normale (16") et grande normale (64"). La petite, à faible rayon d'investigation, permet de déterminer la résistivité des roches envahies par la boue de forage alors que la grande normale, ayant un rayon d'investigation plus grand, fournit la résistivité dans la zone de transition entre les roches vierges et celles envahies par la boue (Desbrandes, 1968; Serra, 1985; Hearst \& Nelson, 1985; Chapellier,1992; Ellis \& Singer, 2007).

\section{Sismique réflexion}

La sismique réflexion (Lavergne, 1986) permet la prospection du sous-sol en se basant sur le déclenchement d'un ébranlement qui donne naissance à des ondes sonores (Telford et al., 1976). En se propageant, ces ondes rencontrent des surfaces qui les réfléchissent en présence de variations des propriétés acoustiques (densité, élasticité) du sous-sol. Ces surfaces sont appelées des réflecteurs. Des géophones à la surface du sol, détectent les ondes qui remontent en surface. Les signaux obtenus sont convertis sous forme de sections sismiques après plusieurs étapes de traitement (Gardner, 1985; Henry, 1997;

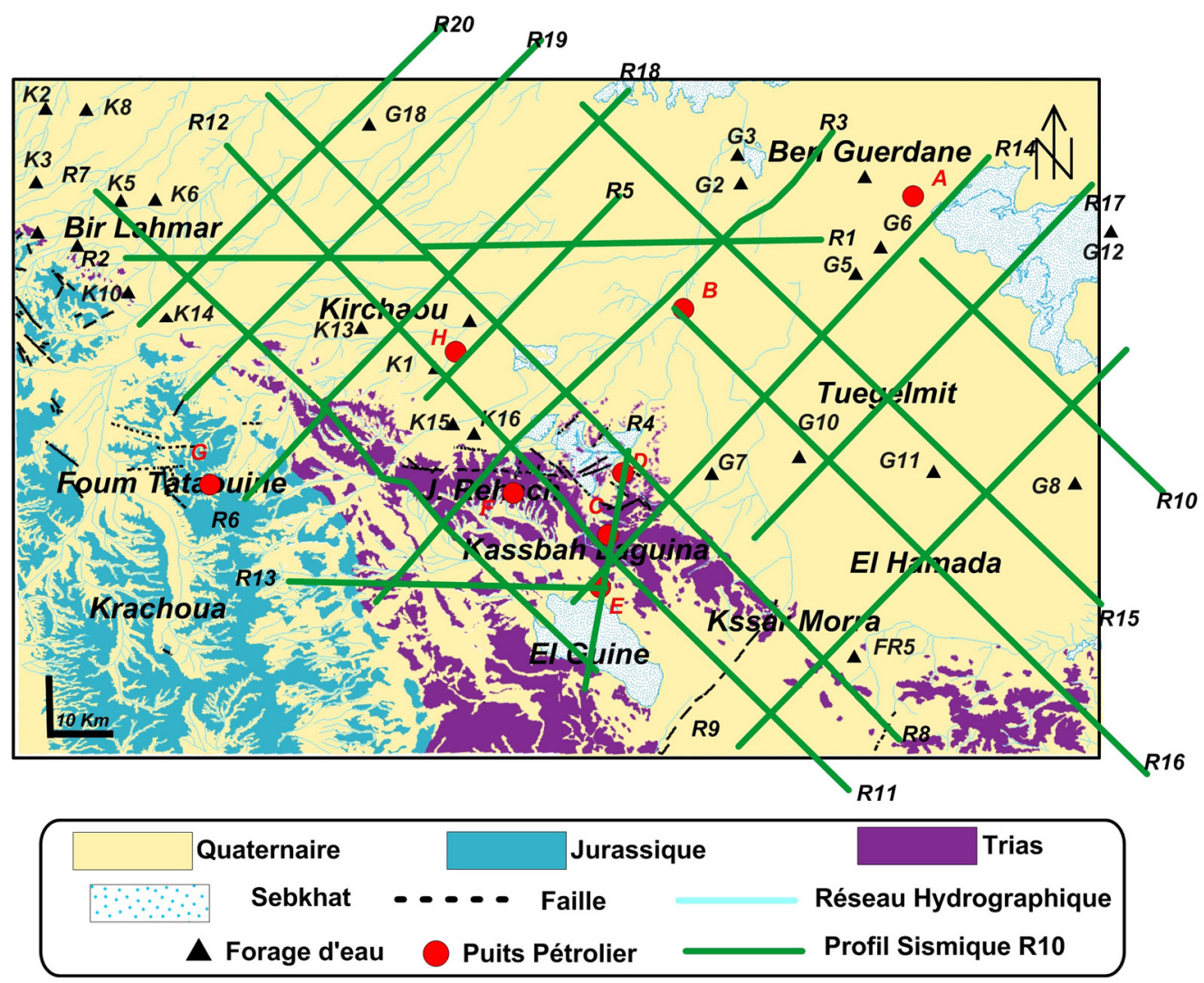

Figure 5.-Carte de répartition des données disponibles. 
Cox, 1999; Inoubli \& Mechler, 1999; Robein, 1999; Mari et al., 2001; Upadhyay, 2004): récupération des amplitudes, corrections statiques, filtrage, analyse de vitesses, corrections dynamiques, sommation et migration.

Vingt profils sismiques ont été interprétés. Ils couvrent l'ensemble du secteur d'étude suivant un maillage homogène. L'interprétation des profils sismiques commence par la sélection des horizons sismiques à pointer. Ces horizons correspondant à des limites stratigraphiques doivent comporter un contraste acoustique suffisant pour produire des réflexions d'amplitude assez élevée, sismiquement caractérisable. Ensuite, on procède au calage sismique. Cette méthode de reconnaissance et de comparaison entre les données de puits et de sismique doit nécessairement comporter au moins les profondeurs des limites stratigraphiques de chaque série ainsi que les relations temps-profondeur. Dans la région de Jeffara-Dahar, les données fournies par les puits pétroliers $\mathrm{H}, \mathrm{C}, \mathrm{D}, \mathrm{B}$ et A sont un secours pour le calage sismique. La conversion des profondeurs en utilisant la courbe temps-profondeur a permis l'identification des différents réflecteurs et particulièrement celui qui correspond à la base de l'aquifère triasique (Fig. 6).

Après l'opération de calage, l'horizon sélectionné est pointé sur les différentes lignes sismiques en insistant sur les fermetures de mailles.
L'interpolation entre les sections sismiques interprétées aboutit à la construction des cartes des isochrones qui exposent les différentes structures tectoniques qui contrôlent la géométrie de l'aquifère du Trias dans la région Jeffara-Dahar.

Les coupes sismiques intégrant l'information géologique et l'interprétation sismique précisent les relations entre les différentes unités hydrogéologiques qui constituent l'aquifère du Trias.

\section{Résultats et interpretation}

\section{Identification des réservoirs triasiques}

L'analyse des colonnes lithologiques et des diagraphies correspondantes (Fig. 7) a permis de déterminer les différentes unités qui composent le système aquifère triasique. Il ressort de cette analyse que les grés de Sidi Stout (Scythien-Anisien), les grés de Kirchaou (Ladinien), les dolomies de Mekraneb (Carnien inférieur), les grés de Touareg (Carnien moyen), et les dolomies de Rehach (Carnien supérieur) constituent des niveaux aquifères intéressants. Les évaporites de Bhir ainsi que les argiles et les évaporites de Mhira sont des aquicludes.

Les logs lithologiques révèlent que la formation Bhir est composée essentiellement d'anhydrites. Les diagraphies (Fig. 7a) détaillent un grand nombre de passages argileux qui n'apparaissent pas

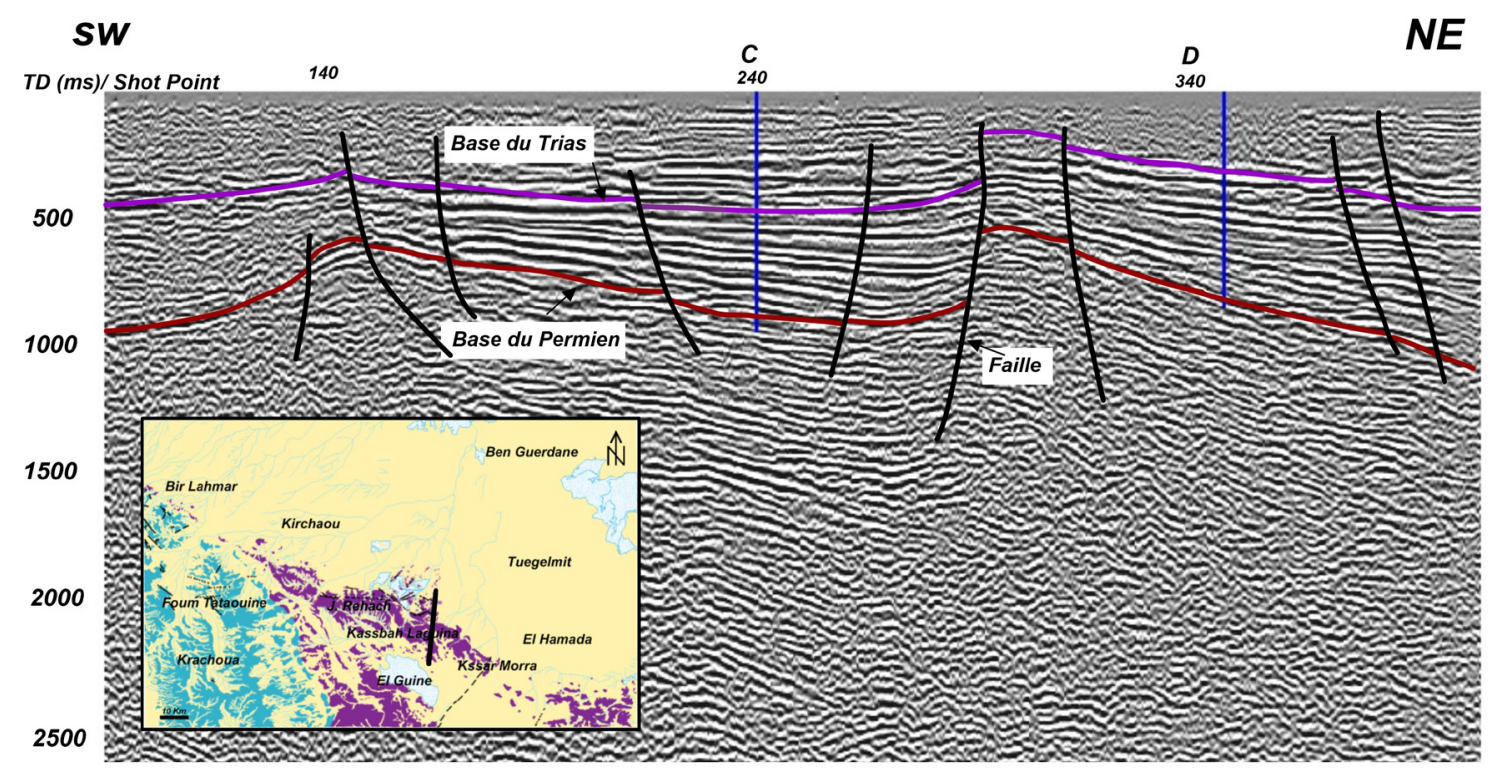

Figure 6.-Calage sismique. 

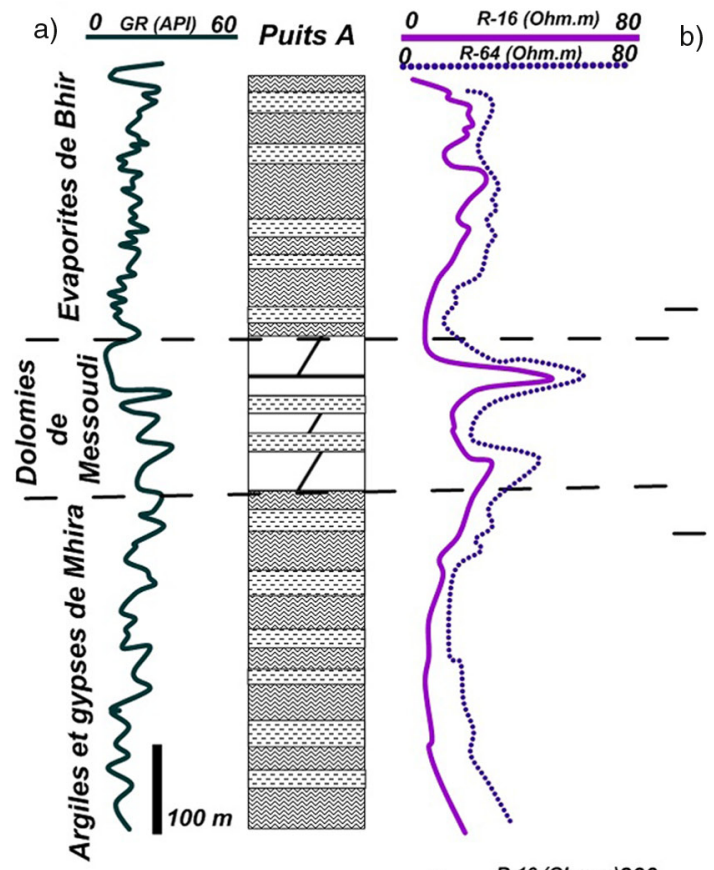

b)
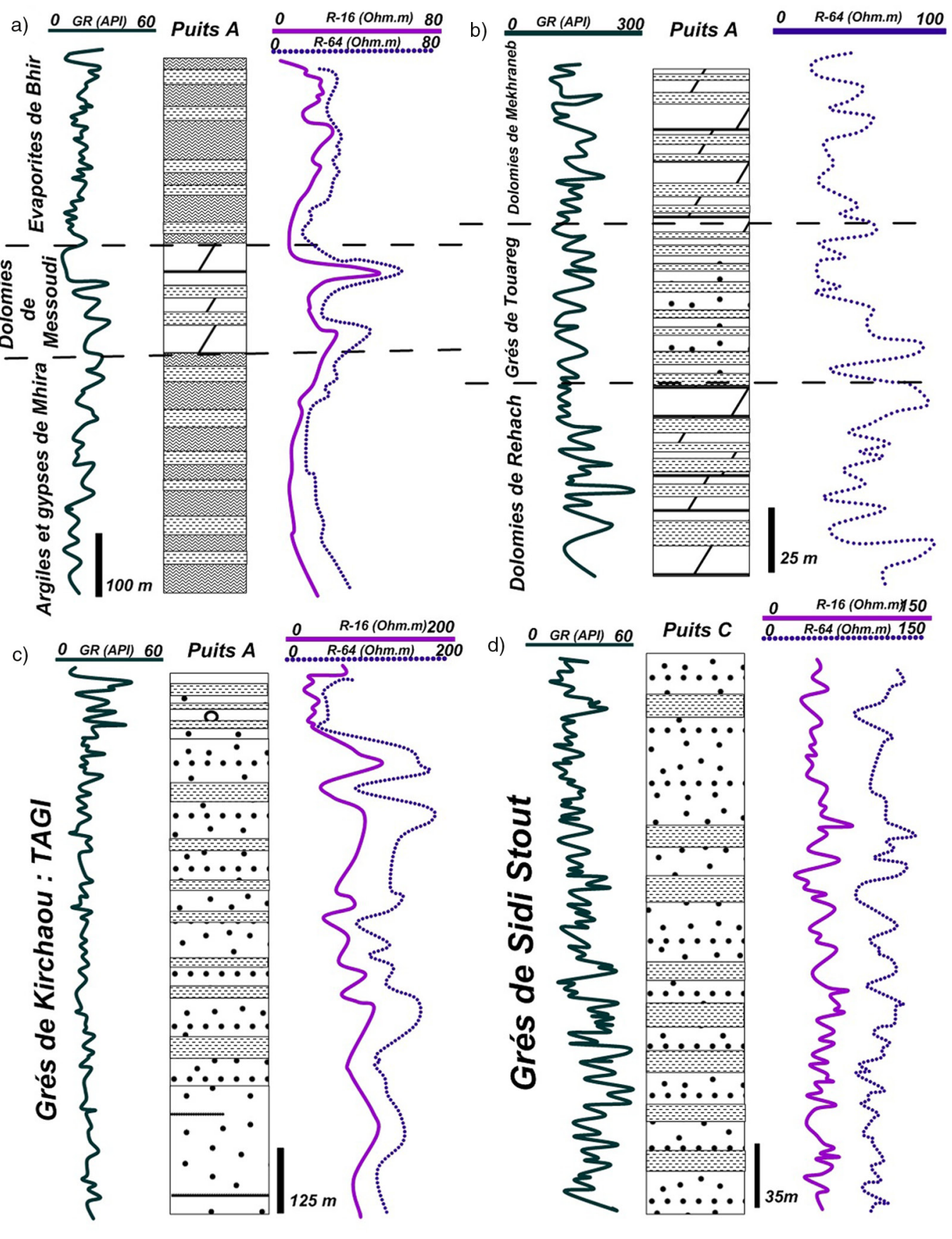

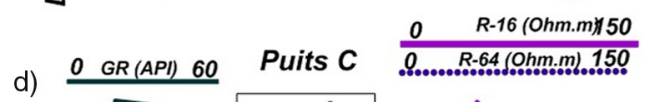
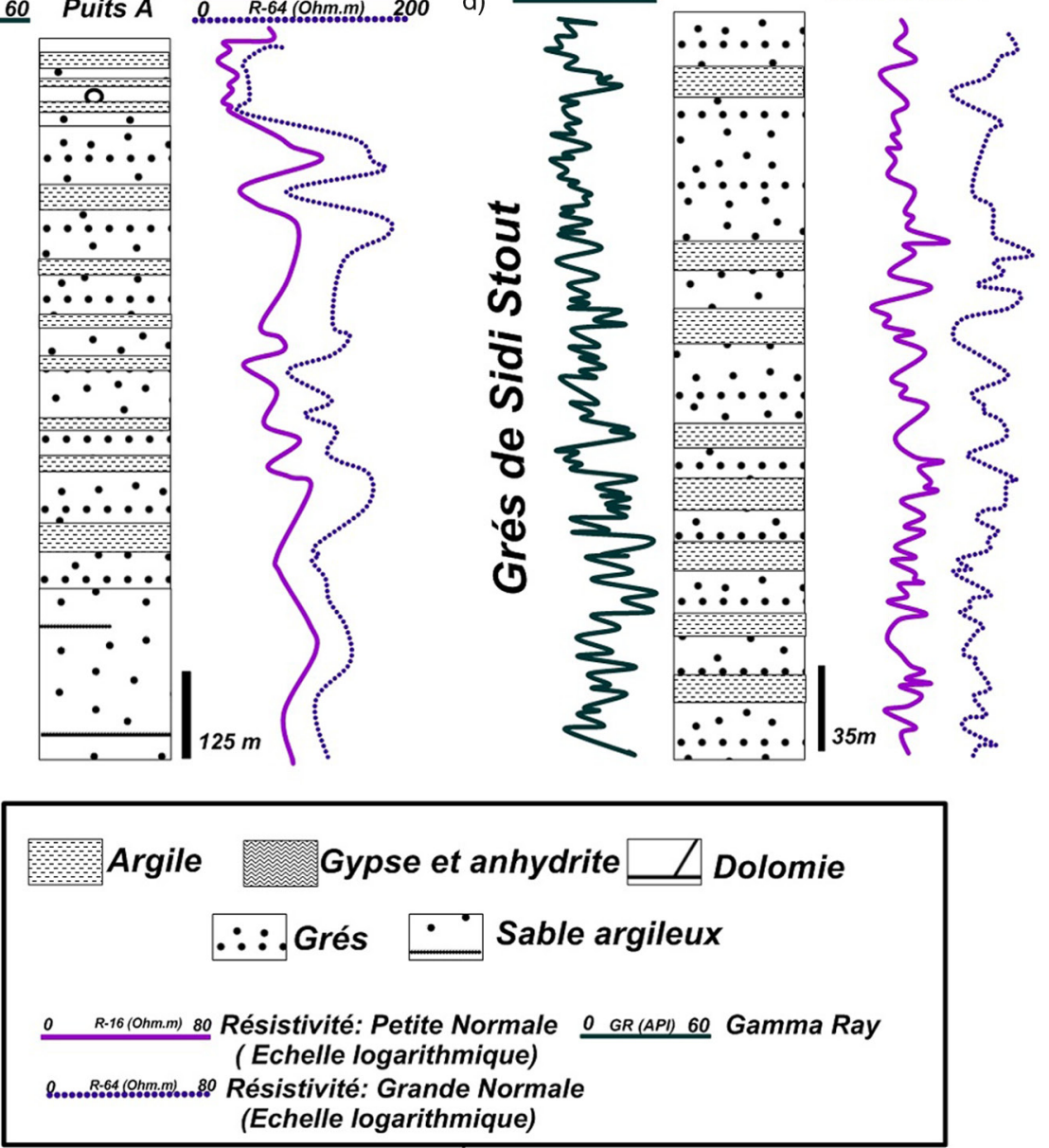

Figure 7.-Caractérisation diagraphique des formations triasiques. 
individuellement sur ces logs. Ils se distinguent par des pics à fortes valeurs de radioactivité naturelle (50 API). Leur résistivité, similaire à celle des évaporites, ne dépasse pas $30 \mathrm{Ohm} \mathrm{m}$.

Egalement, les enregistrements diagraphiques décèlent que les bancs dolomitiques de Messaoudi, caractérisés par des faibles réponses de GR (10 à 30 API) sont intercalés de niveaux d'argiles. Les valeurs de résistivité correspondant à ces bancs sont généralement fortes (50 à $70 \mathrm{Ohm} \mathrm{m}$ ) reflétant un aquifère fracturé d'une bonne qualité d'eau. La faible résistivité du banc médian $(20 \mathrm{Ohm} \mathrm{m})$ peut être attribuée à son épaisseur réduite et par conséquent à l'influence de la réponse des argiles adjacentes. Elle peut être aussi expliquée par l'augmentation de la salinité de l'eau d'imbibition.

Le log Gamma Ray correspondant à la formation Mhira révèle que les pics à faibles valeurs sont les plus développés. Ceci indique que les gypses dominent les argiles dans cette formation. Les mesures de résistivité, homogènes pour l'ensemble, sont de l'ordre de $20 \mathrm{Ohm} \mathrm{m}$.

La formation Azizia (Fig. 7b) est composée par les deux niveaux dolomitiques de Mekraneb et de Rehach séparés par le niveau gréseux de Touareg. La fréquence des intervalles à faible résistivité et à forte radioactivité naturelle traduit la richesse de ces niveaux en argiles.

La formation Kirchaou (Fig. 7c) apparait le réservoir d'eau triasique le plus important. Excepté la partie sommitale riche en argiles, les mesures de radioactivité naturelle enregistrées pour cette formation n'excédant pas 10 API reflétant l'abondance d'éléments grossiers: grés et sables.

Comparé à celui de la formation précitée, le log Gamma Ray de la formation Sidi Stout (Fig. 7d) montre de nombreux intervalles à forte réponse, particulièrement dans la partie basale. Ceci indique des niveaux argileux plus fréquents.

La notable différence de résistivité entre les réservoirs gréseux de Kirchaou et Sidi Stout (60 à 170 Ohm $\mathrm{m}$ ) peut être expliquée par une variation de la salinité de l'eau.

Les corrélations des logs lithologiques, restitués à partir des diagraphies, montrent une importante variation de la profondeur et d'épaisseur des réservoirs triasiques.
La corrélation I (Fig. 8) intégrant les forages d'eau FR1et $\mathrm{H} 1$ ainsi que les puits pétroliers B et A montre l'épaississement des dépôts du Trias de l'Ouest vers l'Est. En effet, dans les forages FR1 et H, ces dépôts sont uniquement représentés par les réservoirs du Trias inférieur (grès de Sidi Stout) d'épaisseur 200 à $220 \mathrm{~m}$. Passant au puits B, $2000 \mathrm{~m}$ des grés du Trias inférieur à moyen (grès de Sidi Stout et grés de Kirchaou) s'intercalent entre les séries permiennes et oligo-miocènes. Au niveau de $\mathrm{A}$, une série triasique complète et épaisse de $3000 \mathrm{~m}$ est recoupée. Les différents réservoirs du Trias supérieur (grés de Touareg et dolomies de Massoudi, Rehach et Mekraneb) et du Trias inférieur à moyen (grés de Sidi Stout et Kirchaou) sont reconnus.

La corrélation II (Fig. 9) relie le forage d'eau FR5 et les puits pétroliers $\mathrm{C}, \mathrm{E}, \mathrm{F}$ et $\mathrm{G}$.

A $573 \mathrm{~m}$ de profondeur, $\mathrm{G}$ a atteint la série triasique composée par la partie basale de l'entité du Trias supérieur, puissante de $85 \mathrm{~m}$ (dolomies de Rehach et de Mekraneb intercalées de grés de Touareg) et les entités du Trias moyen (grès de Kirchaou) et du Trias inférieur (grès de Sidi Stout) respectivement épaisses de $177 \mathrm{~m}$ et $195 \mathrm{~m}$. F expose la même succession lithologique mais avec un net exhaussement des toits, augmentation de l'épaisseur des réservoirs du Trias supérieur $(125 \mathrm{~m})$ et moyen $(292 \mathrm{~m})$ et diminution de celle des grès de Sidi Stout d'âge Trias inférieur $(122 \mathrm{~m})$. Au niveau du E, le Trias supérieur est représenté seulement par $30 \mathrm{~m}$ d'argiles et gypses de Mhira; les réservoirs attribués à cette période sont absents. Dans ce puits l'entité gréseuse de Kirchaou est épaisse de $130 \mathrm{~m}$ alors que celle de Sidi Stout dépasse $650 \mathrm{~m}$. Vers C, les dépôts du Trias supérieur disparaissent et ceux du Trias inférieur et moyen sont plus développés. FR5 n'a recoupé que $235 \mathrm{~m}$ du Trias moyen.

\section{Structuration de l'aquifère du Trias de la Jeffara-Dahar}

La carte des isochrones de la base des dépôts triasiques de la région de Jeffara-Dahar reflète les grands traits structuraux qui caractérisent ces dépôts. Elle montre une succession d'effondrements soulignée par l'existence des dépressions, situées entre 1000 et 2200 ms (Fig. 10). Ces structures, installées dans la partie nord-orientale du secteur d'étude, 


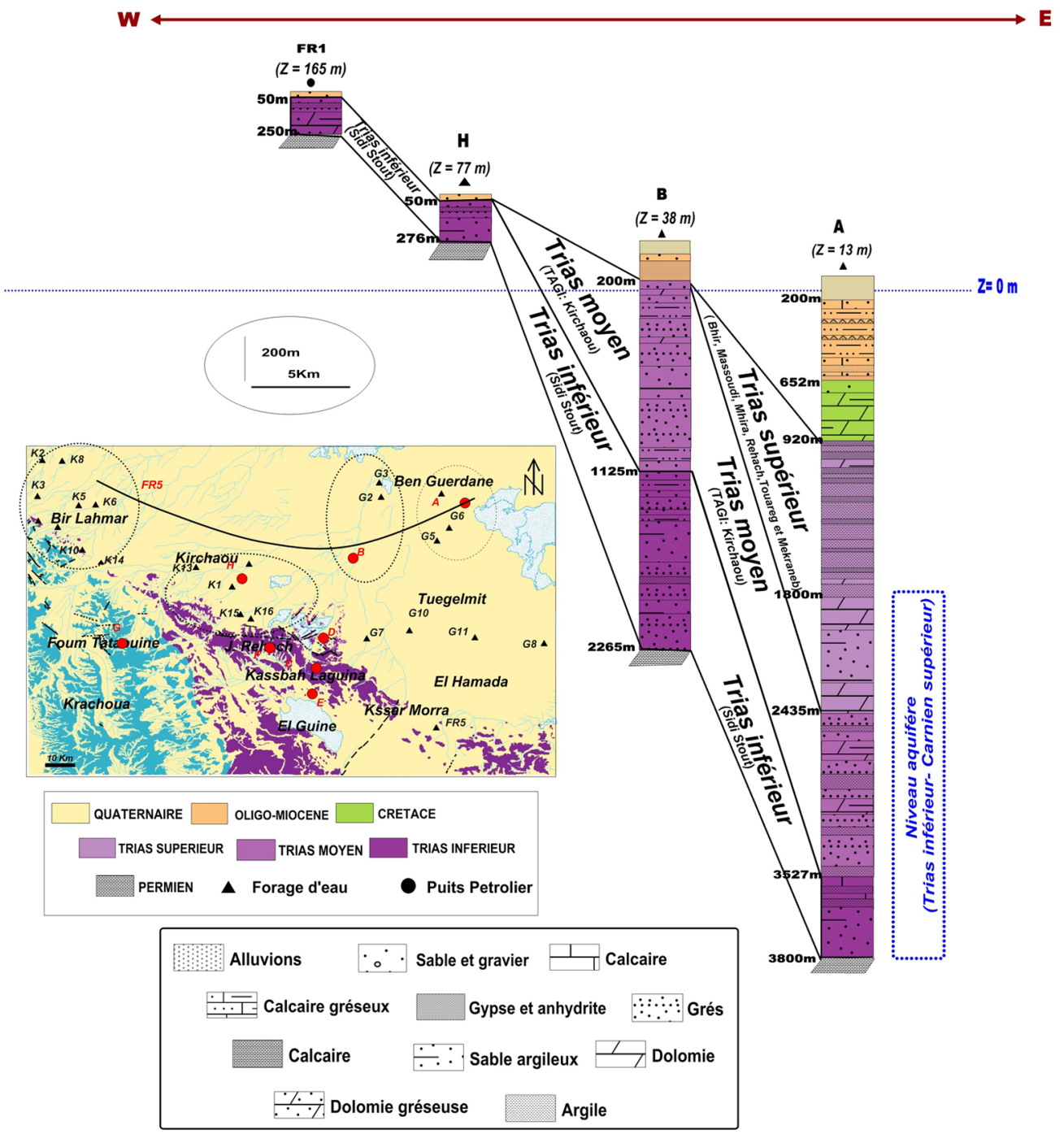

Figure 8.-Corrélation lithologique suivant une direction E-W.

dans la région de Ben Guerdane, sont délimitées par des accidents majeurs à jeu normal et de direction NW-SE.

Des accidents E-W à NE-SW affectent la partie centrale sans générer une remarquable variation de la profondeur de la base du Trias, repérée entre 500 et $600 \mathrm{~ms}$.

Les études géodynamiques (Busson, 1972, Ben Ayed \& Viguier 1981, Zargouni et al., 1985, Bouaziz, 1995) révèlent que trois familles d'accidents majeurs, de directions respectives NE-SW, E-W et NW-SE, ont contrôlé la sédimentation mésozoïque. Dans notre zone d'étude, ce sont les sections sismiques NE-SW qui montrent clairement le plus d'accidents.
Ceci peut s'expliquer par le fait que les failles E-W et NW-SE sont les plus développées (Fig. 11).

La carte structurale du secteur de la Jeffara-Dahar ainsi établie présente les principaux traits hauts et bas et les failles majeures qui caractérisent les séries $\mathrm{du}$ Trias. Ces informations impliquent qu'en plus des axes structuraux déjà décrits sur la carte géologique du secteur, il existe en subsurface des structures plus importantes, qui devront être prises en considération pour comprendre le fonctionnement des systèmes hydrogéologiques.

Les résultats obtenus mettent en évidence l'influence de la tectonique sur la géométrie de l'aquifère triasique dans la région de Jeffara-Dahar; la 


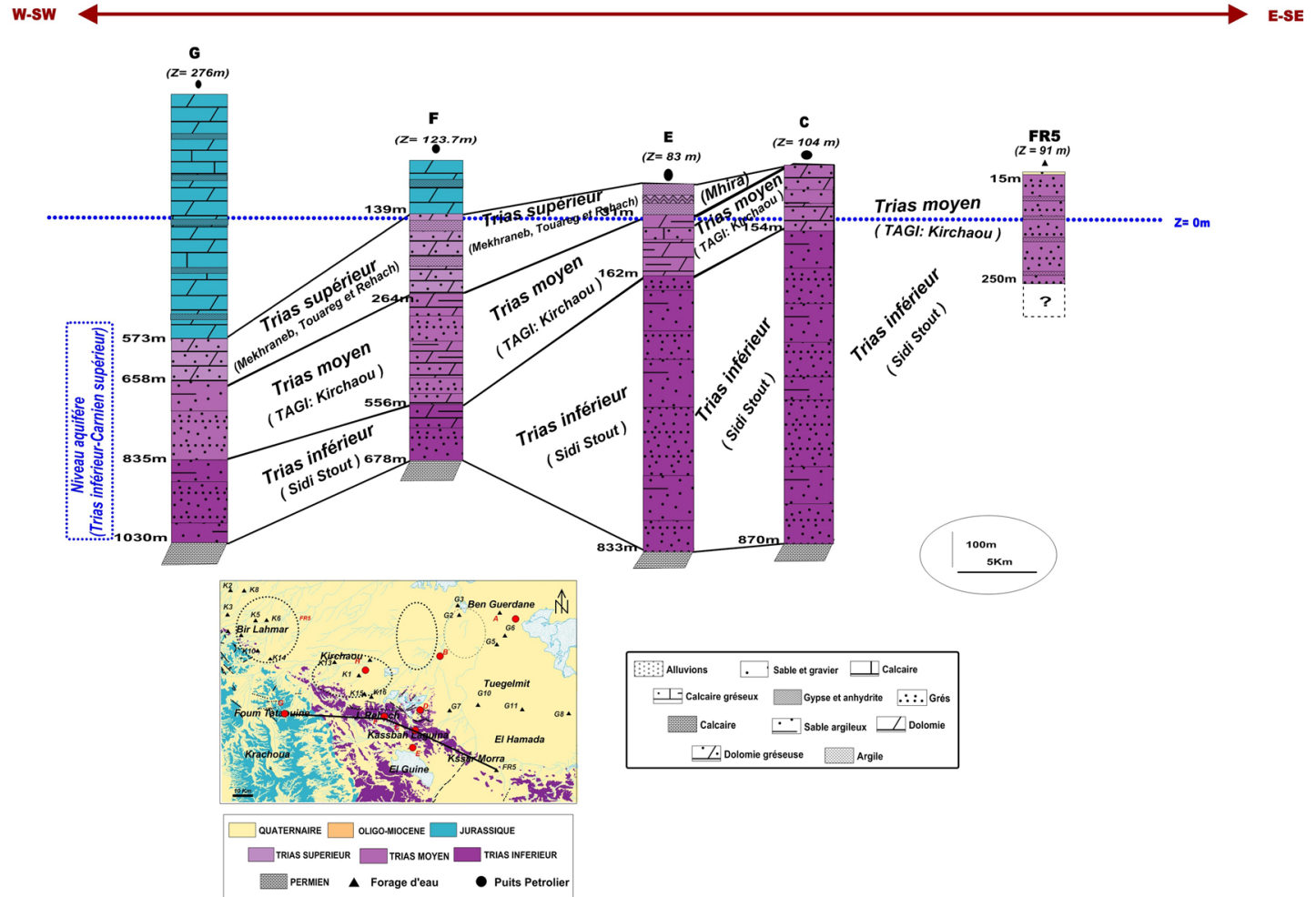

Figure 9.-Corrélation lithologique suivant une direction WSW-ESE.

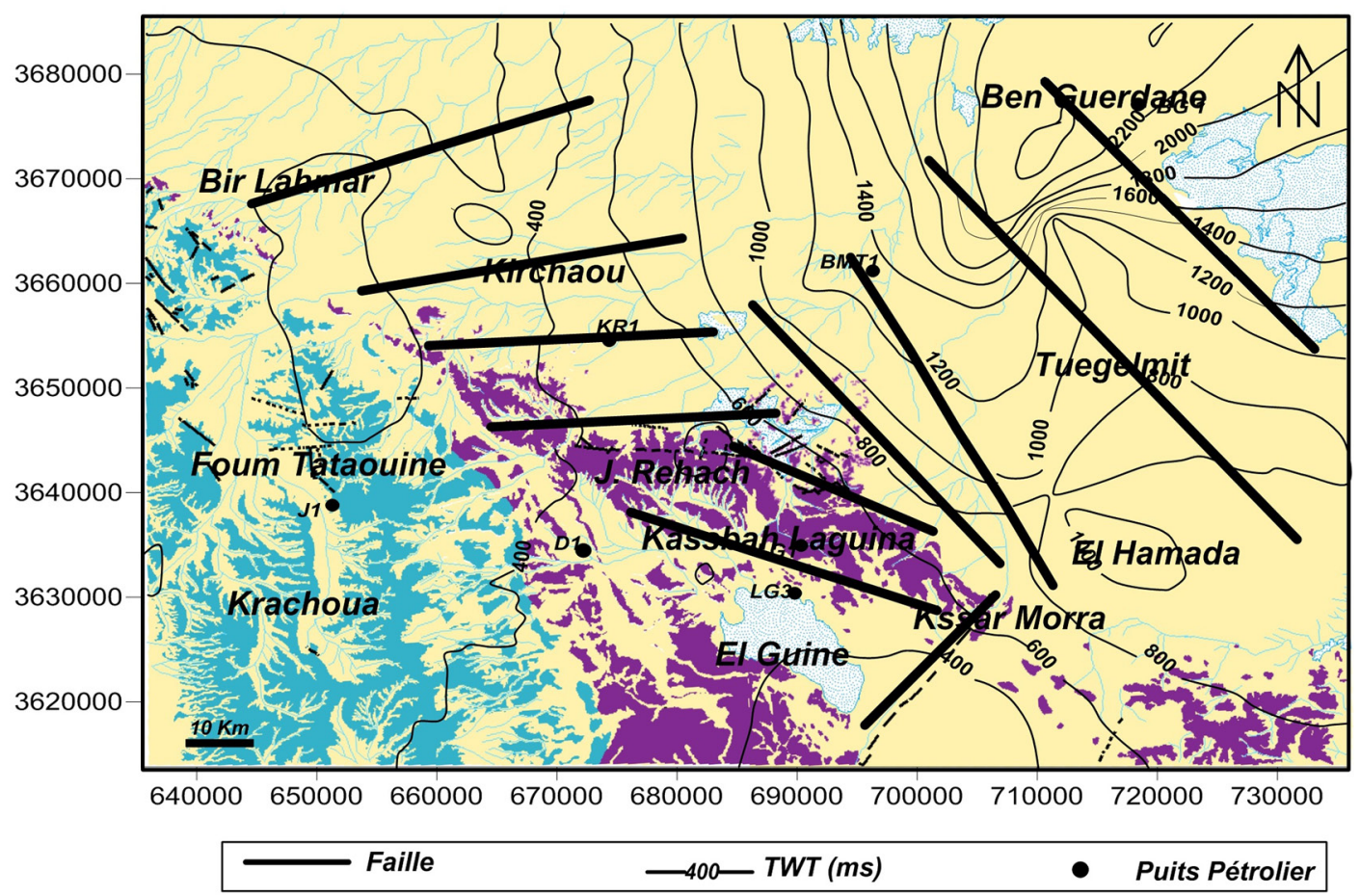

Figure 10.-Carte des isochrones de la base des séries triasiques. 


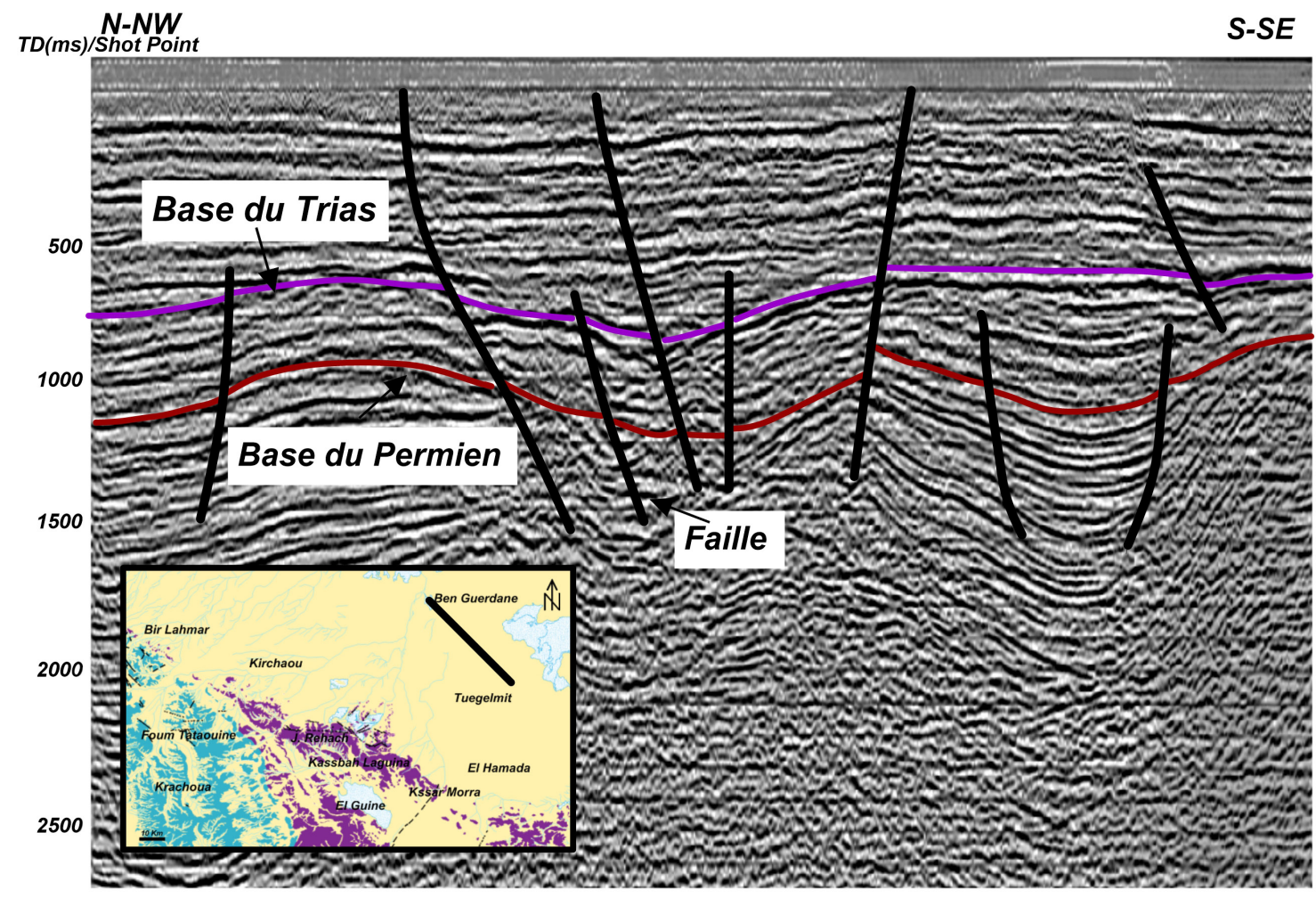

Figure 11.-Profil R10 montrant les grands traits structuraux caractérisant la région.

réactivation des accidents NW-SE, E-W et NE-SW en failles normales sont à l'origine de la compartimentation de cet aquifère en blocs tiltés.

Les coupes sismiques révèlent que les implications d'une telle structuration sont variables d'un secteur à l'autre de la région de Jeffara-Dahar.

La coupe sismique correspondante aux deux profils sismiques R11 et R12 et intégrant les forages d'eau $\mathrm{K} 13$ et $\mathrm{K} 16$ et les puits pétroliers $\mathrm{H}$ et $\mathrm{C}$ (Fig. 12) permet de déceler les relations entre les unités hydrogéologiques dans le secteur KirchaouFoum Tataouine -Sidi Toui. Elle montre que les réservoirs du Trias moyen et inférieur, généralement couverts par les dépôts oligo-miocènes, expriment une notable variation de profondeur et d'épaisseur entre les structures répétitives en horst et graben caractérisant le secteur.

Les puits pétroliers $\mathrm{H}$ et $\mathrm{C}$ sont implantés sur des horsts. Les niveaux aquifères du Trias moyen sont absents dans le premier et affleurent à l'emplacement du second. Ceux du Trias inférieur sont respectivement atteints à $250 \mathrm{~m}$ et $150 \mathrm{~m}$ de profondeur. Également, à l'extrémité Sud-Est de la coupe, la région de Sidi Toui qui correspond à une zone soulevée exhibe des aquifères rehaussés et peu puissants.

Dans la région de Kirchaou, au niveau du forage d'eau K13, exécuté au niveau d'une zone affaissée, les réservoirs du Trias moyen et inférieur sont recoupés à la cote $750 \mathrm{~m}$. Ils s'approfondissent d'avantage tout en allant vers le Nord-Ouest. Au centre de la coupe, dans le forage d'eau $\mathrm{K} 16$, ces réservoirs révèlent leur maximum d'épaisseur $(900 \mathrm{~m})$ reflétant ainsi le jeu synsédimentaire des failles normales.

Par ailleurs, cette coupe révèle une communication latérale entre les différents compartiments malgré les différences de profondeur et d'épaisseur des réservoirs. La similitude des caractéristiques de l'aquifère (piézomètrie et salinité de l'eau) dans les régions de Kirchaou, Foum Tataouine et Sidi Toui traduit cette communication.

La coupe géosismique G2 est établie au profit des profils sismiques R1 et R2 (Fig. 13). G3 correspond au profil R9 (Fig. 14). Elles couvrent respectivement les secteurs Sidi Toui-Ben Guerdane et Kirchaou- Ben Guerdane. Elles montrent que le système aquifère dans ces secteurs est essentiellement 


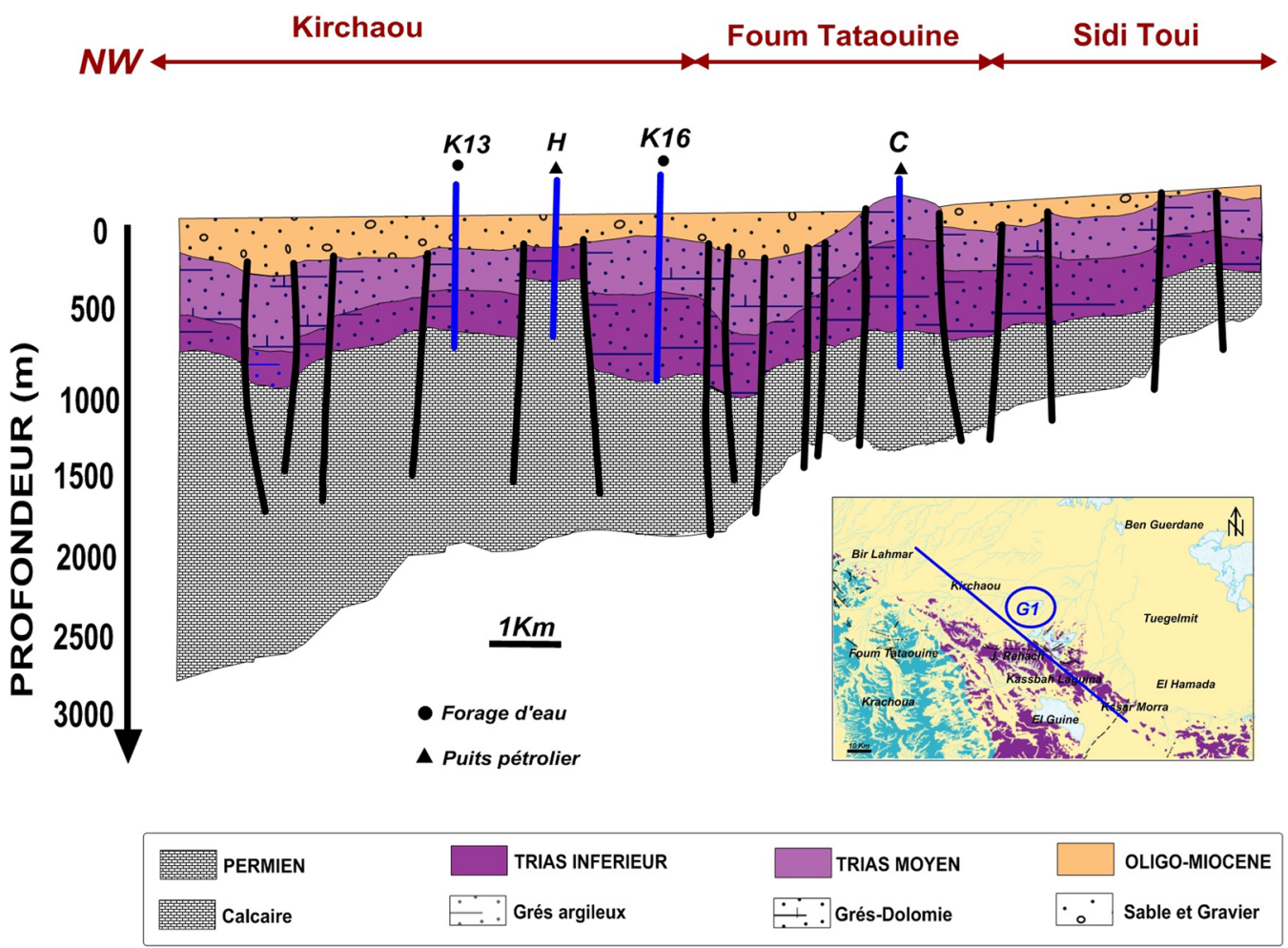

Figure 12.-Coupe sismique G1: NW-SE.

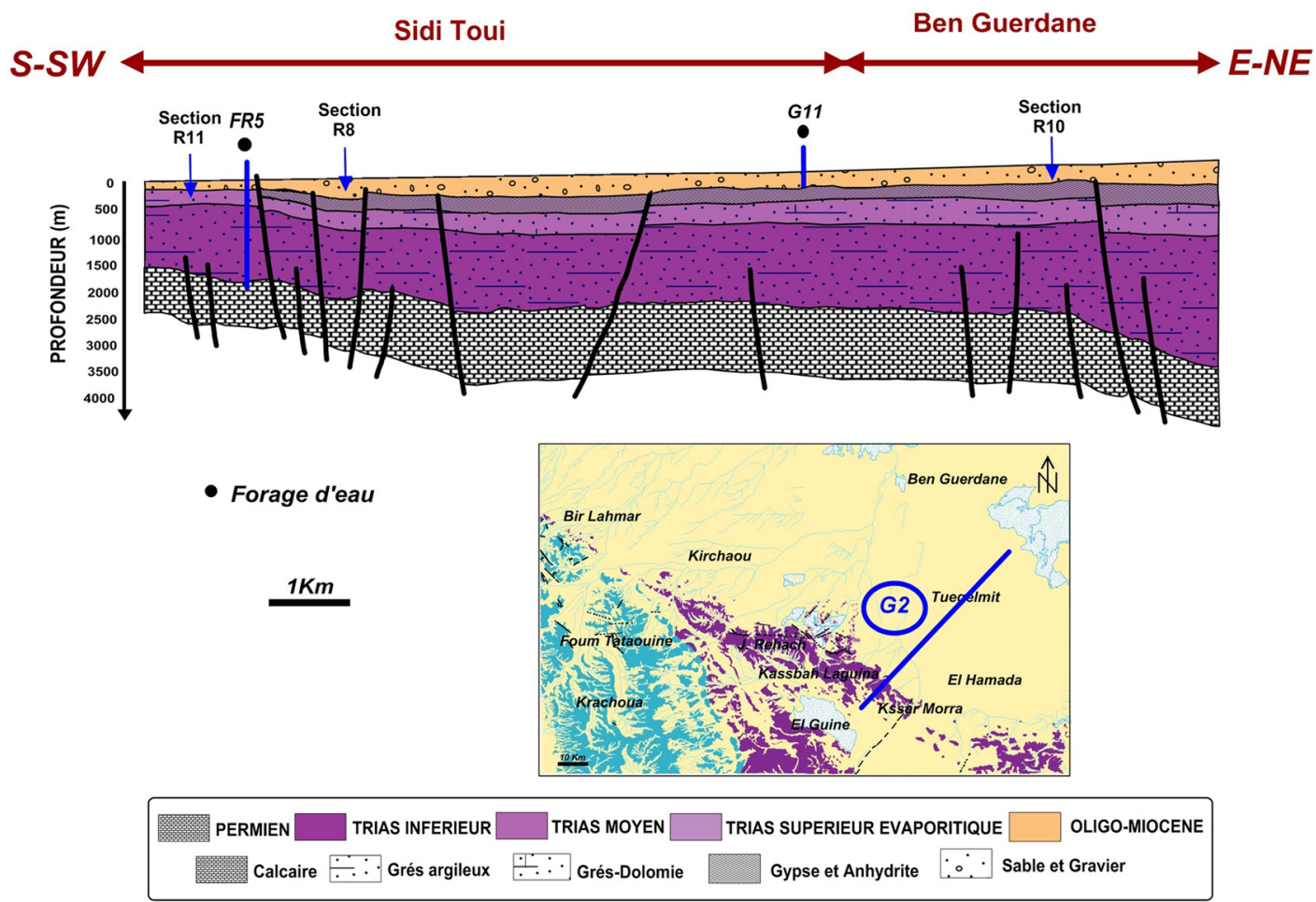

Figure 13.-Coupe sismique G2: SSW-ENE. 


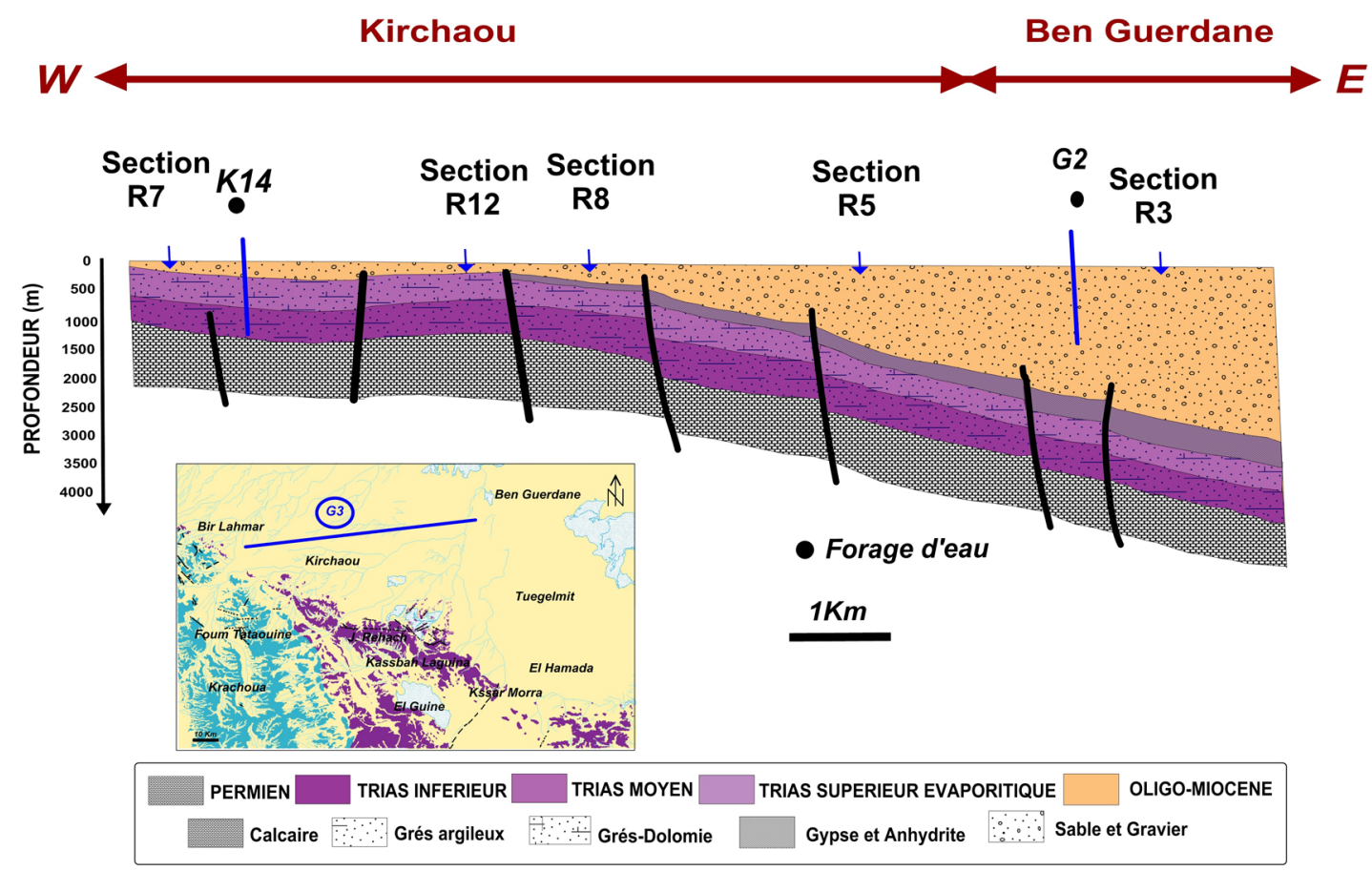

Figure 14.-Coupe sismique G3: W-E.

surmonté par les évaporites du Trias supérieur. L'exception est enregistrée au niveau de Kirchaou et Sidi Toui, à l'extrémité méridionale de G2 et occidentale de G3, où les dépôts oligo-miocènes assurent la couverture.

Il ressort aussi de ces coupes que le jeu normal d'une succession des failles majoritairement orientées NW-SE a généré une notable augmentation de profondeur (Fig. 13 et Fig. 14) et d'épaisseur (Fig. 13) des réservoirs en allant de Kirchaou et Sidi Toui vers Ben Guedane qui correspond à un bassin subsident au cours du Trias. De surcroit l'affaissement progressif des séries perméables dans la structuration en demi-graben résultante a favorisé un écoulement des eaux souterraines vers la partie nord-orientale du secteur d'étude, tel que décrit par la carte piézométrique (Fig. 3).

\section{Conclusion}

La présente étude basée sur les sections sismiques et les données de puits: colonnes lithologiques et diagraphies, décèle les phénomènes sédimentaires et tectoniques qui affectent l'aquifère triasique dans le secteur de Jeffara-Dahar.
L'analyse des colonnes lithologiques et des enregistrements diagraphiques montre que les grés de Sidi Stout (Scythien-Anisien), les grés de Kirchaou (Ladinien), les dolomies de Mekraneb (Carnien inférieur), les grés de Touareg (Carnien moyen), et les dolomies de Rehach (Carnien supérieur) constituent les niveaux aquifères. Les corrélations des colonnes lithologiques montrent une notable variation de la profondeur et de l'épaisseur de ces niveaux.

L'interprétation des sections sismiques montre des structures en horst, graben et demi-graben générées par des failles normales de direction NW -SE à E-W. Ces structures ont compartimenté l'aquifère triasique en blocs soulevés et affaissés. Cette géométrie a d'importantes implications en contrôlant la profondeur, l'épaisseur et l'écoulement des eaux souterraines.

Cette étude présente également un exemple concret d'utilisation de différentes méthodes pour la caractérisation des systèmes aquifères. Dans la reconstitution lithologique, les enregistrements diagraphiques constituent un riche support pour préciser la composition des séries sédimentaires et pour délimiter les intervalles aquifères. La sismique réflexion calée par les données de surface et celles de puits s'avère 
pertinente pour déceler les structures en subsurface et pour la compréhension de la géométrie et du fonctionnement des aquifères.

\section{Références}

Acheche, M.H.; M'Rabet, A.; Ghariani, H.; Ouahchi, A. \& Montgomery, S.L. (2001). Ghdames Basin, southern Tunisia: a reappraisal of Triassic reservoirs ad future prospectivity. AAPG Bulletin, 85: 765-780. http://dx.doi.org/10.1306/8626C9F1-173B-11D7$8645000102 \mathrm{C} 1865 \mathrm{D}$

Abid, K. (2010). Identification et caractérisation hydrogéologique et géochimique de la nappe du Turonien du Sud Tunisien et sa relation avec les aquifères adjacents. Thèse de Doctorat, Ecole Nationale d'Ingénieurs de Sfax, Tunisie, $191 \mathrm{pp}$.

Alem, N.; Assassi, S.; Benhebouche, S.; \& Kadi, B. (1998). Controls on hydrocarbon occurrence and productivity in the F6 reservoir, Tin Fouyé-Tabankort area, NW Illizi Basin. Geological Society, London, Special Publications, 132: 175-186. http://dx.doi.org/10.1144/ GSL.SP.1998.132.01.10

Astier, J.L. (1971). Géophysique appliquée à l'hydrogéologie. Masson, Paris. 277 pp.

Asfahani, J. (2006). Geoelectrical investigation for characterizing the hydrogeological conditions in semiarid region in Khanasser valley, Syria. Journal of Arid Environments, 68:31-52.http://dx.doi.org/10.1016/j. jaridenv.2006.03.028

Baird, D.W. (1967). The Permo-Carboniferous of southern Tunisia. In: Martin, L. (Ed.). Guidebook to the Geology and History of Tunisia. Petroleum Exploration Society of Libya, $9^{\text {th }}$ Annual Field Conference, 85-107.

Ben Ayed, N. \& Viguier, C. (1981). Interprétation structurale de la Tunisie atlasique. Comptes Rendus de l'Academie des Sciences de Paris, Série II., 292: 5-20.

Ben Ayed, N. \& Khessibi, M. (1981). Sur l'évolution géodynamique de la Jeffara tunisienne au cours du Mésozoïque. Notes du Service Géologique (Tunisie), 47: 41-54.

Ben Chelbi, M.; Melki, F. \& Zargouni, F. (2006). Mode de mise en place des corps salifères dans l'Atlas septentrional de Tunisie. Exemple de l'appareil de Bir Afou. Comptes Rendus Geoscience, 338: 349-358. http://dx.doi.org/10.1016/j.crte.2006.02.009

Ben Ismail, H. (1991). Les bassins mésozoïques (Trias à Aptien) du Sud de la Tunisie: Stratigraphie intégrée, Caractéristiques géophysiques et Evolution géodynamique. Thèse de Doctorat, Université de Tunis II, $446 \mathrm{pp}$.

Berkaloff, E. (1933). Contribution à l'étude géologique de l'extrême Sud Tunisien. Le territoire militaire des Matmatas. Bulletin de la Société Géologique de France, 3: 83-85.
Besbes, M. \& Ben Ammar, S. (1985). Représentativité du modèle d'un grand système aquifère: La nappe du Continental Intercalaire du Sahara algéro -tunisien. In: Congrès AMSE, Monastir, Tunisie.

Bobier, C.; Viguier, C.; Chaari, A. \& Chine, A. (1991). The post-Triassic sedimentary cover of Tunisia: Seismic sequences and structure. Tectonophysics, 195: 371-410. http://dx.doi.org/10.1016/0040-1951 (91)90222-E

Bouaziz, S. (1995). Etude de la tectonique cassante dans la plate-forme et l'Atlas sahariens (Tunisie méridionale): Evolution des paleochamps de contraintes et implications géodynamiques. Thèse de doctorat, Université de Tunis II, 485 pp.

Brayard, A.; Escarguel, G. \& Bucher, H. (2007). The biogeography of Early Triassic ammonoid faunas: Clusters, gradients, and networks. Geobios, 40: 749-765. http://dx.doi.org/10.1016/j.geobios.2007.06.002

Burrolet, P-f. (1963). Discussion sur la stratigraphie libyenne. Revue de l'Institut Francais du Pétrole, 18(10): 1323-1329.

Busson, G. (1970). Le Mésozoïque saharien: 2ème partie: Essai de synthèse des données des sondages algérotunisiens. CNRS, Paris, 811 pp.

Busson, G. (1972). Principes, méthodes et résultats d'une étude stratigraphique du Mésozoique Saharien. Mémoires du Muséum National d'Histoire Naturelle - Série C, 26. 441 pp.

Castany, G. (1954). L'accident Sud-tunisien, son âge et ses relations avec l'accident Sud -atlasique de l'Algérie. Comptes Rendus de l'Academie des Sciences de Paris, 238: 916-918.

Chalbaoui, M. \& Ben Dhia, H. (2004). Principales caractéristiques des réservoirs du sud-ouest tunisien. Comptes Rendus de l'Académie des Sciences de Paris, 336: 1191-1198.

Chaouachi, M.Ch. (1988). Etude sédimentologique des séries du Permien supérieur du J. Tebaga de Medenine, Sud-Est de la Tunisie: Genèse, Diagenèse et potentiel du réservoir des corps récifaux. Thèse 3 ème cycle, Université de Tunis, 299 pp.

Chapellier, D. (1992). Well logging in hydrogeology. A.A. Balkema Publishers, Brook field, MA, 175 pp.

Chikhaoui, M.; Jallouli, C.; Turki, M.C.; Soussi, M.; Braham, A. \& Zaghbib-Turki, D. (2002). L'affleurement triasique du Debadib-Ben Gasseur (Nord-Ouest de la Tunisie): diapir enraciné à épanchements latéraux dans la mer Albienne, replissé au cours des phases de compression tertiaires. Comptes Rendus Geoscience, 334: 1129-1133. http://dx.doi.org/ 10.1016/S1631-0713(02)01856-4

Cox, M. (1999). Static corrections for seismic reflection surveys. Society of Exploration Geophysicists, $546 \mathrm{pp}$.

Cuif, J.P. \& Gautret, P. (1987). Comparaison des modalités de diagenèse du squelette de Spongiaires carbonatés dans le Trias de Turquie et le Permien de Tunisie. 
Geobios, 20: 757-773. http://dx.doi.org/10.1016/ S0016-6995(87)80003-7

Desbrandes, R. (1968). Théorie et interprétation des diagraphies. Institut Français du Pétrole, 547 pp.

DGRE. (2012). Annuaire pluviométrique de la Tunisie, 2013. Direction Générale des Ressources en eaux.

Duvernoy, B. (1994). Tunisian and pelagian basin (Africa Exploration Opportunities; Vol.13). Proceedings of the $4^{\text {th }}$ Tunisian petroleum Exploration Conference. Tunis.

Ellis, D.V. \& Singer, J.M. (2007). Well logging for earth scientists. Springer, Berlin, 708 pp. http://dx.doi.org/ 10.1007/978-1-4020-4602-5

Espitalié, J.; Maxwell, J.R.; Chenet, Y. \& Marques, F. (1988). Aspects of hydrocarbon migration in the Mesozoic in the Paris Basin as deduced from an organic geochemical survey. Organic Geochemistry, 13: 467-481. http://dx.doi.org/10.1016/0146-6380(88)90068-X

Gabtni, H.; Alyahyaoui, S.; Jallouli, C.H.; Hasni, W. \& Mickus, K. (2012). Gravity and seismic reflection imaging of a deep aquifer in an arid region: Case history from the Jeffara basin, southeastern Tunisia. Journal of African Earth Sciences, 66-67: 85-97. http://dx.doi.org/10.1016/j.jafrearsci.2012.03.007

Gand, G.; Vianey-Liaud, M.; Demathieu, G. \& Garric, J. (2000). Deux nouvelles traces de pas de Dinosaures du Trias supérieur de la bordure cévenole (la GrandCombe, Sud-Est de la France). Geobios, 33: 599-624. http://dx.doi.org/10.1016/S0016-6995(00)80033-9

Gardner, G.H.F. (1985). Migration of seismic data. Society of Exploration Geophysicists, 462 pp.

Garrach, M. (2005). Contribution à l'étude hydrogéologique de la nappe du calcaire Bathonien à Tataouine, exploitation et perspectives. Mémoire de DEA en Géologie appliquée, Université de Carthage, Tunis.

Gasmi, M. (2002). Apports de la géophysique à la reconnaissance et la gestion des ressources naturelles. Application en Tunisie. Thèse de Doctorat, Université de Sfax, 471 pp.

Gautret, P. (1991). Présence d'un Stromatopore calcitique dans le Trias de Turquie (nappes d'Antalya, Alakir Cay). Geobios, 24: 417-421. http://dx.doi. org/10.1016/S0016-6995(06)80240-8

Gharbi, R.A.; Chihi, L.; Hammami, M.; Abdelkader, S. \& Kadri, A. (2005). Manifestations tectono-diapiriques synsédimentaires et polyphasées d'âge Crétacé supérieur-Quaternaire dans la région de Zag Et Tir (Tunisie centre-nord). Comptes Rendus Geoscience, 337: 1293-1300. http://dx.doi.org/10.1016/j. crte.2005.07.006

Glintzboechkel, C.H. \& Rabate, J. (1964). Microfaunes et microfaciès du Permo -Carbonifère du Sud tunisien. Leiden: Brill, 45 pp.

Gouasmia, M.; Gasmi, M.; Mhamdi, A.; Bouri, S. \& Ben Dhia, H. (2006). Prospection géoélectrique pour l'étude de l'aquifère thermal des calcaires récifaux, Hmeïma-Boujabeur (Centre ouest de la Tunisie).
Comptes Rendus Geosciences, 338: 1219-1227. http://dx.doi.org/10.1016/j.crte.2006.09.017

Guellala, R.; Inoubli, M.H. \& Amri, F. (2009). Nouveaux éléments sur la structure de l'aquifère superficiel du Ghardimaou (Tunisie). Hydrological Sciences Journal, 54: 974-983. http://dx.doi.org/10.1623/hysj. 54.5.974

Guellala, R.; Ben Marzoug, H.; Inoubli, M.H. \& Moumni, L. (2011). Apports de la sismique réflexion à l'étude de l'aquifère du Continental Intercalaire du Jérid (Tunisie). Hydrological Sciences Journal, 56: 1040-1052. http://dx.doi.org/10.1080/02626667.2011.590809

Guellala, R.; Abidi, M.; Balti N.; Inoubli, M.H.; Tagorti, M.A. \& Amri, F. (2014). Prospection géophysique pour la recherche d'eau souterraine dans le Nord-Ouest de la Tunisie. Hydrological Sciences Journal. http://dx.doi.org/10.1080/02626667.2014.914213

Hartmann, M. (1963). Einige geochemische Untersuchungen an Sandsteinen aus Perm und Trias. Geochimica et Cosmochimica Acta, 27: 459-499. http://dx.doi. org/10.1016/0016-7037(63)90084-X

Hearst, J.R. \& Nelson, PH. (1985). Well logging for physical properties. New York: Mc Graw-Hill, 570 pp.

Henry, G. (1997). La sismique réflexion, principes et développements. Éditions TECHNIP, 172 pp.

Hoa, T.T.; Anh, T.T.; Phuong, N.T.; Dung, P.T.; Anh, T.V.; Izokh, A.E.; Borisenkob, A.S.; Lanc, C.Y.; Chungd, S.L. \& Lod, C.H. (2008). Permo-Triassic intermediate-felsic magmatism of the Truong Son belt, eastern margin of Indochina. Comptes Rendus Geoscience, 340(2-3): 112-126. http://dx.doi. org/10.1016/j.crte.2007.12.002

Inoubli, M.H. \& Mechler, P. (1999). Expression géologique et apport du champ de vitesse de sommation dans l'amélioration de la résolution sismique. Notes du Service Géologique de Tunisie. 54 pp.

Jaffal, M.; Kchikach, A.; Lefrod, J.P. \& Hanich, L. (2002). Contribution à l'étude d'une partie du bassin d'Essaouiria (Maroc) par sismique réflexion. Comptes Rendus Géosciences, 334: 229-234. http://dx.doi. org/10.1016/S1631-0713(02)01756-X

Jallouli, C.; Chikhaoui, M.; Braham, A.; Turki, M.M.; Mickus, K. \& Benassi, R. (2005). Evidence for Triassic salt domes in the Tunisian Atlas from gravity and geological data. Tectonophysics, 396: 209-225. http://dx.doi.org/10.1016/j.tecto.2004.12.003

Jamal, M.; Bizra. Y. \& Caron, C. (2000). Palaeogeography and hydrocarbon habitat of the Triassic series in Syria. Comptes Rendus de l'Académie des Sciences Séries IIA, 331: 133-139. http://dx.doi.org/10.1016/ S1251-8050(00)01389-6

Kamoun, F.; Peybernès, B.; Cizak, R. \& Calzada, S. (2001). Triassic paleogeography of Tunisia. Palaeogeography, Palaeoclimatology, Palaeocology, 172: 223-242. http://dx.doi.org/10.1016/S0031-0182(01)00283-8

Khessibi, M. (1985). Etude sédimentologique des affleurements permiens du Djbel Tebaga de Médenine 
(Sud Tunisien). Bulletin du Centre de recherches Exploration Production Elf-Aquitaine, 9: 427-464.

Khomsi, S.; Bédir, M.; Ben Jemia, M.G. \& Zouar, I.H. (2004). Mise en évidence d'un nouveau front de chevauchement dans l'Atlas tunisien oriental de Tunisie par sismique réflexion. Contexte structural régional et rôle du Trias salifère. Comptes Rendus Geoscience, 336: 1401-1408. http://dx.doi.org/10.1016/j. crte.2004.06.007

Koussoubé, Y.; Nakolendoussé, S.; Bazié, P. \& Savadogo, A.N. (2003). Typologie des courbes des sondages électriques verticaux pour la reconnaissance des formations superficielles et leur incidence en hydrogéologie de socle cristallin du Burkina. Sud Sciences et Technologies, 10: 26-32.

Lachaal, F.; Bedir, M.; Tarhouni, J.; Gacha, A.B. \& Leduc, C. (2011). Characterizing a complex aquifer system using geophysics, hydrodynamics and geochemistry: a new distribution of Miocene aquifers in the Zeramdine and Mahdia-Jebeniana blocks (eastcentral Tunisia). Journal of African Earth Sciences, 60: 222-236. http://dx.doi.org/10.1016/j.jafrearsci. 2011.03 .003

Larroque, F. \& Dupuy, A. (2004). Apports de la méthode sismique réflexion à l'identification des structures profondes des formations tertiaires en Médoc (Gironde, France): Implications hydrogéologiques. Comptes Rendus Géosciences, 336: 1111-1120. http:// dx.doi.org/10.1016/j.crte.2004.04.009

Lavergne, M. (1986). Méthodes sismiques. Editions TECHNIP et l'Institut Francais de Petrole, 207 pp.

Lemaire, M.M.; Westphal, M.; Montigny, R.; Gurevitch, E.L.; Feinberg, H.; Pozzi, J-P. \& Nazarov, K. (1998). Paléomagnétisme et évolution du bloc Scythe-Touran du Permien inférieur au Trias supérieur. Comptes Rendus de l'Académie des Sciences Series IIA, 327: 441-448. http://dx.doi.org/10.1016/ S1251-8050(99)80071-8

Li, N.; Chen, Y.-J.; Fletcher, I.R. \& Zeng, Q.-T. (2011). Triassic mineralization with Cretaceous overprint in the Dahu Au-Mo deposit, Xiaoqinling gold province: Constraints from SHRIMP monazite $\mathrm{U}-\mathrm{Th}-\mathrm{Pb}$ geochronology. Gondwana Research, 20: 543-552. http://dx.doi.org/10.1016/j.gr.2010.12.013

Mamou, A. (1990). Caractéristiques, Evolutions et Gestion des Ressources en Eau du Sud tunisien. Thèse de Doctorat Es-Sciences, Université de Paris, 426 pp.

Mansouri, M. (1988). Extrême sud tunisien, étude hydrogéologique du continental Intercalaire. Tome 1: Géologie, hydrologie et géométrie du réservoir du continental intercalaire. Direction des études. Société nationale d'exploitation et de distribution des eaux, $54 \mathrm{pp}$.

Mari, J.L.; Glangeaud, F.C. \& Coppens, F. (2001). Traitement du signal pour géologues et géophysiciens. Tome 2: Techniques de base. Institut Français de Pétrole, 288 pp.
Mathieu, G. (1949). Contribution à l'étude des Monts Troglodytes dans l'Extrême Sud -tunisien. Annales des mines et de la géologie, 4, 82 pp.

Meyer de Stadelhofen, C. (1991). Applications de la géophysique aux recherches d'eau. Paris: Tech. Doc. Lavoisier, $192 \mathrm{pp}$.

M'Rabet, A.; Chaouachi, M.C.; Razghallah, S. \& Duvernoy, B. (1987). The upper Permian carbonate and siliciclastic series of Jebel Tebaga (Southern Tunisia): an example of platform to slop sedimentation. $8^{\text {th }}$ IAS Regional Meeting of Sedimentology (Tunis), Abstracts, p. 262-263.

Naouali Sarsar, B.; Guellala, R. \& Inoubli, M.H. (2013). Gravity Data Analysis for Hydrocarbon Prospection: Subsalt Structures in Mateur Area (Diapir Zone, Northern Tunisia). AAPG/SEG exploration of subsult structures in rift basins workshop. Jordan.

Newell, N.D.; Rigbuy, J.K.; Driggs, A.; Boyd, O.W. \& Stehli, F.G. (1976). Permian reef complex, Tunisia. Brigham Young University Geology Studies, 23(1):75-112.

Oliver, G.; Zaw, K.; Hotson, M.; Meffre, S. \& Manka, T. (2014). U-Pb zircon geochronology of Early Permian to Late Triassic rocks from Singapore and Johor: A plate tectonic reinterpretation. Gondwana Research, 26: 132-143. http://dx.doi.org/10.1016/j. gr.2013.03.019

OSS (2003). Système Aquifère du Sahara Septentrional. Volume 2: Hydrogéologie. Projet SASS. Rapport interne. Direction Génerale des Ressources en Eau, Tunis, $275 \mathrm{pp}$.

Ould Baba Sy, M. (2005). Recharge et paléorecharge du système aquifère du Sahara Septentrional. Thèse Doctorat, Université de Tunis II, 261 pp.

Perthuisot, V. (1983). Diapirism in Northern Tunisia. Journal of Structural Geology, 3: 231-235. http://dx. doi.org/10.1016/0191-8141(81)90019-5

Peybernes, B.; Kamoun, F.; Ben Youssef, M.; Trigui, A.; Ghanmi, M.; Zarbout, M. \& Frechengues, M. (1993). Sequence stratigraphy and micropaleontology of the Triassic series from the southern part of Tunisia. Journal of African Earth Sciences (and the Middle East), 17: 293-305. http://dx.doi.org/10.1016/08995362(93)90074-Z

Piñeiro, G.; Ramos, A. \& Marsicano, C. (2012). A rhinesuchid-like temnospondyl from the PermoTriassic of Uruguay. Comptes Rendus Palevol, 11: 65-78. http://dx.doi.org/10.1016/j.crpv.2011.07.007

Raulin, C.; Lamotte, D.F.; Bouaziz, S.; Khomsi, S.; Mouchot, N.; Ruiz, G. \& Guillocheau, F. (2011). Late Triassic-early Jurassic block tilting along E-W faults, in southern Tunisia: New interpretation of the Tebaga of Medenine. Journal of African Earth Sciences, 61: 94-104. http://dx.doi.org/10.1016/j. jafrearsci.2011.05.007

Razgallah, S.; Chaouachi, M. \& M'Rabet, A. (1989). Les récifs à algues du Permien supérieur du Jebel Tebaga 
de Mednine. Sud -Est de la Tunisie. Géologie Méditerranéenne, 15: 213-231.

Rebeix, R.; Le Gal La Salle, C.; Michelot, J-L.; Verdoux, P.; Noret, A.; Monvoisin, G.; Gianesinni, S.; Lancelot, J. \& Simler, R. (2011). Tracing the origin of water and solute transfers in deep groundwater from Oxfordian, Dogger and Trias formations in the east of the Paris Basin - France. Physics and Chemistry of the Earth, Parts A/B/C, 36: 1496-1510. http://dx.doi.org/ 10.1016/j.pce.2011.07.015

Robein, E. (1999). Vitesse et techniques d'imagerie en sismique réflexion: principes et méthodes. Paris: Lavoisier, $380 \mathrm{pp}$.

Roger, F.; Jolivet, M. \& Malavieille, J. (2008). Tectonic evolution of the Triassic fold belts of Tibet. Comptes Rendus Geoscience, 340: 180-189. http://dx.doi. org/10.1016/j.crte.2007.10.014

Rossi, Ph.; Cocherie, A.; Fanning, C.M. \& Ternet, Y. (2003). Datation UPb sur zircons des dolérites tholéiitiques pyrénéennes (ophites) à la limite TriasJurassique et relations avec les tufs volcaniques dits « infra-liasiques 》 nord-pyrénéens. Comptes Rendus Geoscience, 335: 1071-1080. http://dx.doi. org/10.1016/j.crte.2003.09.011

Serra, O. (1985). Diagraphies différées. Bases de l'interprétation. Tome 2, Interprétation des données diagraphiques. Bulletin des centres de Recherches Exploration -Production Elf Aquitaine, 7.

Sumanovac, F. (2006). Mapping of thin sandy aquifers by using high-resolution reflection seismics and 2-D electrical tomography. Journal of Applied Geophysics, 58: 144-157. http://dx.doi.org/10.1016/j.jappgeo.2005. 06.005

Telford, W.M.; Gedrart, L.P.; Sherriff, R.E. \& Key, D.A. (1976). Prospection géophysique. Tome 1, Prospection sismique. Cambridge University, 258 pp.

Termier, H.; Termier, G. \& Vachard, D. (1977). Monographie paléontologique des affleurements permiens du Djbel Tebaga (Sud -Tunisien). Stuttgart: Schweizerbart, 109 pp.
UNESCO (1972). Etude des Ressources en Eau du Sahara Septentrional (Algérie et Tunisie). Rapport sur les résultats du projet (Conclusions et recommandations).

Upadhyay, S.K. (2004). Seismic reflection processing: with special reference to anisotropy. Springer, $636 \mathrm{pp}$. http://dx.doi.org/10.1007/978-3-662-09843-1

Vila, J.M.; Ben Youssef, M.; Bouhlel, S.; Ghanmi, M.; Kassa, S. \& Miaadi, F. (1998). Tectonique en radeaux au toit d'un "glacier de sel" sous-marin albien de Tunisie du Nord-Ouest: exemple du secteur minier de Gueurn Halfaya. Comptes Rendus de l'Académie des Sciences, Séries IIA, 327: 563-570. http://dx. doi.org/10.1016/S1251-8050(99)80039-1

Yahyaoui, H. (1996). Etude hydrogéologique des aquifères du Piémont Oriental et du flanc Occidental du Dahar (Régions de Remada et de Déhibat). Thèse Doctorat, Faculté de Sciences de Tunis, 171 pp.

Zammouri, M. (1990). Contribution à une révision des modèles hydrogéologiques du Sud tunisien. Thèse de Doctorat, Universite Tunis II, Tunisie.

Zargouni, F.; Rabia, C.H. \& Abbes, C.H. (1985). Rôle des couloirs de cisaillement de Gafsa et de Negrine -Tozeur dans la structuration du faisceau des plis des chotts, éléments de l'accident Sud-Atlasique. Comptes Rendus de l'Academie des Sciences de Paris, Série II, 301: 831-834.

Zouhri, L.; Gorni, CH.; Lamouroux, CH.; Vachard, D. \& Dakki, M. (2003). Intérprétation hydrogéologique de l'aquifère des bassins sud -rifains (Maroc): apport de la sismique réflexion. Comptes Rendus Géosciences, 335: 319-326. http://dx.doi.org/10.1016/ S1631-0713(03)00052-X

Zouhri, L.; Gorini, C.; Mania, J.; Deffontaines, B. \& Zerouali, A. (2004). Spatial distribution of resistivity in the hydrogeological systems, and identification of the catchment area in the Rharb basin, Morocco. Hydrological Sciences Journal, 49: 387-398. http:// dx.doi.org/10.1623/hysj.49.3.387.54350 